\title{
An energy-preserving muscle tissue model: formulation and compatible discretizations
}

\author{
D. Chapelle†, P. Le Tallec $\ddagger$, P. Moireau†, M. Sorine† \\ $\dagger$ INRIA, Rocquencourt, BP 105, 78153 Le Chesnay cedex, France \\ $\ddagger$ Ecole Polytechnique, 91128 Palaiseau cedex, France
}

Published in Journal for Multiscale Computational Engineering 10(2):189-211 (2012)

\begin{abstract}
In this paper we propose a muscle tissue model - valid for striated muscles in general, and for the myocardium in particular - based on a multi-scale physiological description. This model extends and refines an earlier-proposed formulation by allowing to account for all major energy exchanges and balances, from the chemical activity coupled with oxygen supply to the production of actual mechanical work, namely, the biological function of the tissue. We thus perform a thorough analysis of the energy mechanisms prevailing at the various scales, and we proceed to propose a complete discretization strategy - in time and space - respecting the same balance laws. This will be crucial in future works to adequately model the many important physiological - normal and pathological - phenomena associated with these energy considerations.
\end{abstract}

Keywords: muscle tissue modeling; myocardium; multiscale; energy balance; time and space discretizations

\section{Introduction}

The modeling of the active mechanical behavior of muscle tissues - and of the myocardium in particular - has been the object of an abundant literature, see for example $[11,19,27,10,18$, $31,16]$ and references therein, with some detailed experimental validations in most instances - at the local (tissue) or global (organ) levels, or both, see e.g. [6, 12]. Of course, in order to adequately model the muscle physiology, energy considerations - balances and exchanges - are of utmost value, and such mechanisms are well-described at the cellular level [15]. However, little attention has so far been devoted to carrying over these considerations to the macroscopic level in the muscular tissue models, whereas key phenomena such as adaptation to effort and oxygen consumption - and correspondingly pathologies associated with shortage of oxygen supply, such as ischemia and infarction - critically depend on energy mechanisms.

The main objective in this paper is to revisit a muscular tissue formulation based on the constitutive law proposed in [4], with its incorporation in a three-dimensional nonlinear continuum mechanics framework discussed and assessed in [31] - see also $[26,8,6]$ - in order to

- refine and more extensively establish the global validity of this approach as regards energy exchange mechanisms, from the microscopic to the macroscopic levels;

\footnotetext{
${ }^{*}$ Corresponding author: dominique.chapelle@inria.fr
} 
- propose some adequate discretization strategies - in time and space - which carefully preserve these energy exchanges.

Note that in the above-mentioned references this formulation was primarily proposed and assessed to model the heart behavior, but it is in fact valid for all types of striated muscles - namely, cardiac or skeletal muscles.

An outline of the paper is as follows. In Section 2 we briefly recall the derivation of the fundamental laws of continuum mechanics, primarily to introduce the notation used in the paper. Then we describe the proposed muscular tissue formulation, starting from the multi-scale modeling of the myofibres in Section 3, and proceeding with the complete model formulation in Section 4, with a detailed analysis of the energy mechanisms prevailing at the various scales. Finally, in Section 5 we propose and analyze some adequate time and space discretization strategies derived to preserve the previously established energy mechanisms, before giving some concluding remarks in Section 6.

\section{Notation and modeling framework}

In this section we sketch the derivation of the fundamental law of dynamics - and the associated principle of virtual work - in a total Lagrangian framework, with the primary objective of introducing the required notation.

\section{$2.1 \quad$ Kinematics}

We consider a deformable solid which occupies at time $t$ the space domain $\Omega(t)$ - denoted by $\Omega$ when there is no ambiguity- with boundary $\partial \Omega(t)$. The total Lagrangian formulation consists in describing the position history of each point with respect to a reference configuration $\left(\Omega_{0}, \partial \Omega_{0}\right)$ - not necessarily equal to $(\Omega(0), \partial \Omega(0))$. Thus, the deformation is a one-to-one mapping $\phi$ from the reference configuration to the current configuration providing the position of each point at each time:

$$
\underline{\phi}:\left\{\begin{aligned}
\bar{\Omega}_{0} & \rightarrow \mathbb{R}^{3} \\
\underline{\xi} & \mapsto \underline{x}=\underline{\phi}(\underline{\xi}, t)
\end{aligned}\right.
$$

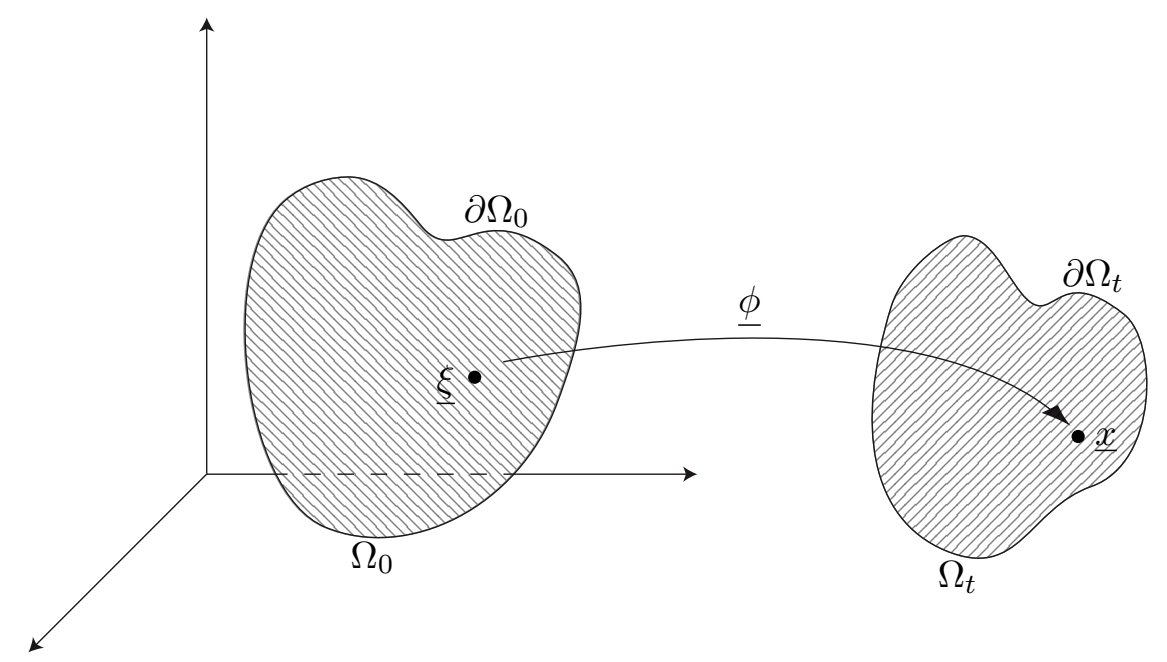

Figure 1: Reference and deformed configurations linked by the deformation map. 
We denote by $y$ the displacement field

$$
\underline{y}(\underline{\xi}, t)=\underline{\mathrm{x}}-\underline{\xi}=\underline{\phi}(\underline{\xi}, t)-\underline{\xi},
$$

and $\underline{\underline{F}}$ is the deformation gradient

$$
\underline{\underline{F}}(\underline{\xi}, t)=\underline{\underline{\nabla}} \underline{\underline{\phi}}=\underline{\underline{\mathbb{1}}}+\underline{\underline{\nabla}}_{\underline{\xi}} \underline{y},
$$

such that the deformed volume is given by $J d \Omega$ where $J=\operatorname{det} \underline{\underline{F}}$ and $d \Omega$ is the volume measure (here in the reference configuration), while a change of area is given by $J \underline{\underline{F}}^{-T} \cdot \underline{d S}$. Furthermore, we introduce the right Cauchy-Green deformation tensor $\underline{\underline{C}}=\underline{\underline{F}}^{T} \cdot \underline{\underline{F}}$. We finally recall that the local changes of geometry are described by the Green-Lagrange strain tensor denoted by $\underline{\underline{e}}$ and defined by

$$
\underline{\underline{e}}=\frac{1}{2}(\underline{\underline{C}}-\underline{\underline{\mathbb{1}}})=\frac{1}{2}\left(\underline{\underline{\nabla}} \underline{\underline{y}}+\left(\underline{\underline{\nabla}}_{\underline{\xi}}\right)^{T}+(\underline{\underline{\nabla}} \underline{\underline{y}})^{T} \cdot \underline{\underline{\nabla}}_{\underline{\xi}} \underline{y}\right)
$$

with linearized expression $\underline{\underline{\varepsilon}}$ given by

$$
\underline{\underline{\varepsilon}}=\frac{1}{2}\left(\underline{\underline{\nabla}} \underline{\underline{y}} \underline{\underline{\underline{\nabla}}}+(\underline{\underline{\nabla}} \underline{\underline{y}})^{T}\right)
$$

\subsection{Fundamental law of dynamics}

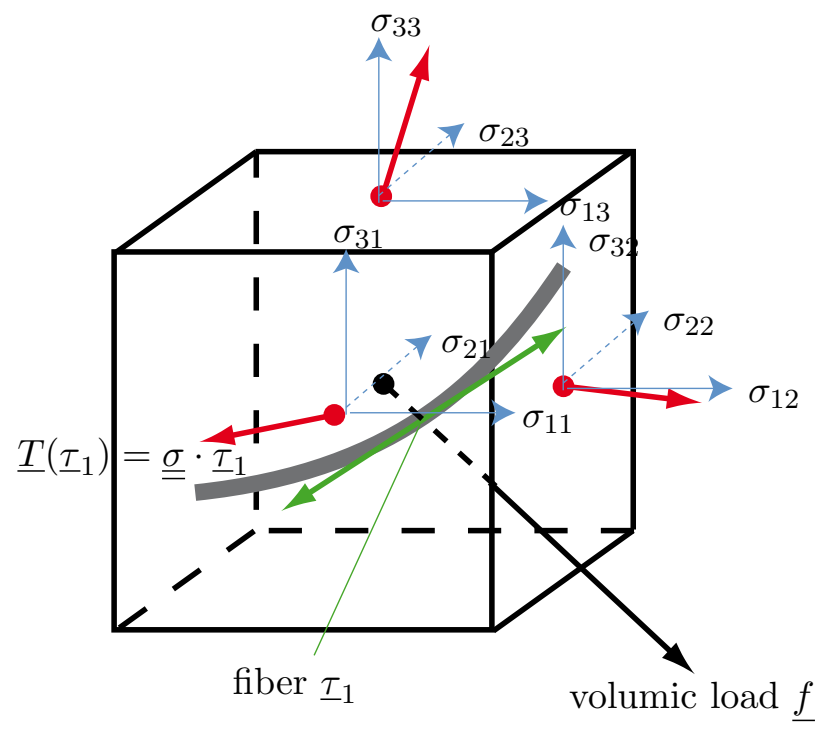

Figure 2: Cauchy cube adapted to muscle tissue. We represent the Cauchy stress tensor $\underline{\underline{\sigma}}$ components and the resulting traction $\underline{T}$ on each surface. The fiber will appear in the complete definition of cardiac constitutive laws in Section 3.

In the deformed configuration, the fundamental law of dynamics coupling the stresses and the external forces can be formulated from the Cauchy cube in Figure 2 as

$$
\underline{\nabla}_{\underline{x}} \cdot \underline{\underline{\sigma}}+\rho(\underline{f}-\underline{\gamma})=0, \text { in } \Omega
$$

where $\underline{\sigma}$ is the Cauchy stress tensor, $\rho$ is the mass per unit volume of the solid, $f$ is the external force per unit mass, and $\underline{\gamma}$ the acceleration. The corresponding weak formulation is given in the space of admissible displacements by

$$
\forall \underline{v}^{*} \in V(\Omega), \quad \int_{\Omega} \underline{\underline{\sigma}}: \underline{\underline{\nabla}}_{\underline{\underline{v}}} \underline{v}^{*} d \Omega=\int_{\Omega} \rho(\underline{f}-\underline{\gamma}) \cdot \underline{v}^{*} d \Omega,
$$


in the absence of boundary tractions. Considering the symmetry of the Cauchy stress tensor, we can symmetrize $\nabla_{\underline{\underline{x}}}$ to introduce $\underline{\underline{\varepsilon}}\left(\underline{v}^{*}\right)=\frac{1}{2}\left(\underline{\nabla}_{\underline{x}} \underline{v}^{*}+\left(\underline{\underline{\nabla}}_{x} \underline{v}^{*}\right)^{T}\right)$, the linearized deformation tensor in the deformed configuration, so that the weak formulation of the fundamental law of dynamics becomes

$$
\forall \underline{v}^{*} \in V(\Omega), \quad \int_{\Omega} \underline{\underline{\sigma}}: \underline{\underline{\varepsilon}}\left(\underline{v}^{*}\right) d \Omega=\int_{\Omega} \rho(\underline{f}-\underline{\gamma}) \cdot \underline{v}^{*} d \Omega
$$

The change of variable $\underline{x} \rightarrow \underline{\xi}$ allows to convert this expression into a total Lagrangian form with respect to the reference configuration. In fact, we have by the chain rule

$$
\underline{\underline{\nabla}}_{\underline{\underline{v}}} \underline{v}^{*}=\underline{\underline{\nabla}}_{\underline{\xi}} \underline{v}^{*} \cdot \underline{\underline{\nabla}} \underline{\underline{\xi}} \underline{\underline{\xi}}=\underline{\underline{\nabla}}_{\underline{\xi}} \underline{v}^{*} \cdot\left(\underline{\underline{\nabla}}_{\underline{\xi}}\right)^{-1}=\underline{\underline{\nabla}}_{\underline{\xi}} \underline{v}^{*} \cdot \underline{\underline{F}}^{-1}
$$

hence we get

$$
\forall \underline{v}^{*} \in V\left(\Omega_{0}\right), \quad \int_{\Omega_{0}} \underline{\underline{\sigma}}:\left(\underline{\underline{\nabla}}_{\underline{\xi}} \underline{v}^{*} \cdot \underline{\underline{F}}^{-1}\right) J d \Omega=\int_{\Omega_{0}} \rho_{0}(\underline{f}-\underline{\gamma}) \cdot \underline{v}^{*} d \Omega
$$

In order to use symmetric tensors defined on the reference configuration, we consider the second Piola-Kirchhoff stress tensor

$$
\underline{\underline{\Sigma}}=\underline{\underline{F}}^{-1} \cdot \underline{\underline{T}}=J \underline{\underline{F}}^{-1} \cdot \underline{\underline{\sigma}} \cdot \underline{\underline{F}}^{-T}
$$

so that the weak formulation of the equation of motion becomes

$$
\forall \underline{v}^{*} \in V\left(\Omega_{0}\right), \quad \int_{\Omega_{0}} \underline{\underline{\Sigma}}:\left(\underline{\underline{F}}^{T} \cdot \underline{\underline{\nabla}}_{\underline{\xi}} \underline{\underline{v}}^{*}\right) d \Omega=\int_{\Omega_{0}} \rho_{0}(\underline{f}-\underline{\gamma}) \cdot \underline{v}^{*} d \Omega .
$$

Then, noting that $\underline{\underline{\nabla}}_{\underline{\underline{v}}} \underline{v}^{*}=\mathrm{d}_{\underline{y}} \underline{\underline{F}} \cdot \underline{v}^{*}-$ by which we denote the differential of $\underline{\underline{F}}$ with respect to the displacement field, applied on $\underline{v}^{*}$ - we infer that the symmetrized version of $\underline{\underline{F}}^{T} \cdot \underline{\underline{\nabla}}_{\underline{\xi}}$ is the derivative of the Green-Lagrange tensor with respect to the displacement

$$
\mathrm{d}_{\underline{y}} \underline{\underline{e}} \cdot \underline{v}^{*}=\frac{1}{2}\left(\left(\mathrm{~d}_{\underline{y}} \underline{\underline{F}} \cdot \underline{v}^{*}\right)^{T} \cdot \underline{\underline{F}}+\underline{\underline{F}}^{T} \cdot \mathrm{d}_{\underline{y}} \underline{\underline{F}} \cdot \underline{v}^{*}\right),
$$

leading to the so-called principle of virtual work formulated on the reference configuration, that we will use from now on

$$
\forall \underline{v}^{*} \in V\left(\Omega_{0}\right), \quad \int_{\Omega_{0}} \rho_{0} \underline{\gamma} \cdot \underline{v}^{*} d \Omega+\int_{\Omega_{0}} \underline{\underline{\Sigma}}: d_{\underline{y}} \underline{\underline{e}} \cdot \underline{v}^{*} d \Omega=\int_{\Omega_{0}} \rho_{0} \underline{f} \cdot \underline{v}^{*} d \Omega .
$$

\section{Remark 1}

The energy balance is classically obtained in solid mechanics using as a test function $\underline{v}^{*}$ in the weak formulation the actual velocity field $\underline{v}=\underline{\dot{y}}$. Therefore, the relation $\underline{\underline{\varepsilon}}(\underline{v})=\underline{\underline{\varepsilon}}$ means that the Cauchy stress tensor $\underline{\underline{\sigma}}$ is the energy conjugate of the deformation tensor $\underline{\underline{\varepsilon}}$. Comparatively, the second Piola-Kirchhoff stress tensor $\underline{\underline{\Sigma}}$ is the energy conjugate of the Green-Lagrange deformation tensor $\underline{\underline{e}}$ since $d_{\underline{y}} \underline{\underline{e}} \cdot \underline{v}=\underline{\dot{e}}$.

\section{Multi-scale modeling of the active myofibres}

We now present a multi-scale modeling approach of the active part of the constitutive law, after a brief anatomical description. 


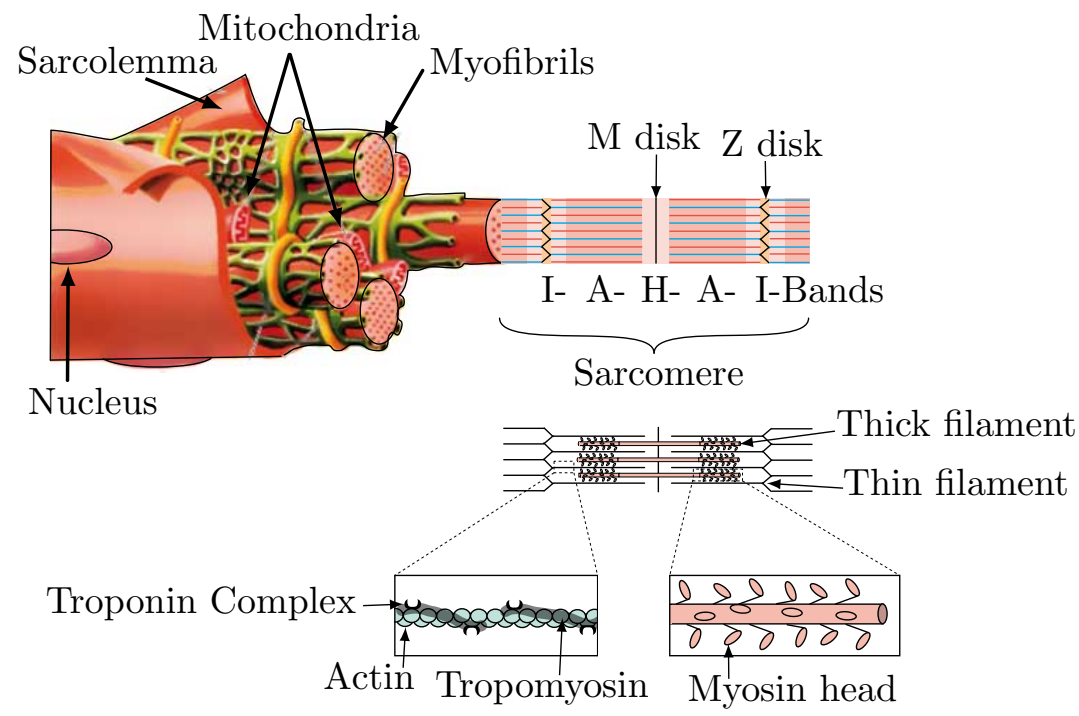

Figure 3: Myofiber description.

\subsection{Anatomical description}

The following description holds for all so-called striated muscles, namely, it is valid both for the cardiac muscle and skeletal muscles.

Striated muscles - illustrated in Fig. 3, are multi-scale structures for which the main component is the muscular cell - also called myocyte. This cell is a fiber of diameter ranging from 10 to $100 \mu \mathrm{m}$ and of length up to $15 \mathrm{~cm}$ for skeletal muscles. A myocyte is circumscribed by a cellular membrane called sarcolemma, which encloses the sarcoplasm - a fluid more generally called cytoplasm in a "generic" cell - with sarcosomes - some specialized mitochondria - and typically about a hundred myofibrils.

A myofibril is divided into compartments of about $2 \mu \mathrm{m}$ in length, called sarcomeres and separated by Z-discs. When observed with a microscope they appear as a succession of bands alternatively light and dark, hence the denomination of "striated muscle". This is in fact due to their structure based on thick filaments made of myosin and thin filaments of actin. Between two successive Z-disks, three different types of bands can be observed:

- the I-band made only of actin filaments (several thousands in each sarcomere);

- the A-band in which the two types of filaments coexist;

- the H-band only composed of myosin filaments, and with the so-called M-disk in the center.

A more detailed analysis of the myosin filament reveals that it is made of a assemblage of about 300 molecules of myosin-II. Each such molecule features two globular heads connected by the "neck region" to the tail domain - this terminology can be understood at first sight in Figure 4. Each myosin head has a force-producing region with a nucleotide-binding pocket for ATP, and a special actin-binding site. It is the swinging motion of the heads interacting with actin which induces the sliding of the filaments along each other.

Concerning actin - a globular molecule - about 400 such molecules can interconnect like a pearl necklace to form a polymer. In fact, as seen in Fig. 4 an actin filament is made of two such polymers side by side associated with tropomyosin molecules wound around them. In addition, every $40 \mathrm{~nm}$ or so a troponin molecule is attached, and this molecule is responsible for interacting with myosin heads. 
Sarcomeres also incorporate an elastic compound of more than $100 \mathrm{~nm}$ in length: titin, which connects Z-disks to M-disks.

The process by which calcium concentration gradients lead to contraction is referred to as the sliding filament mechanism. In a sarcomere actin and myosin filaments are laid out along each other, and myosin heads can bind to actin filaments with a given angle. This bond is called actin-myosin bridge. Due to a structural modification of the myosin-II molecule, this angle can change, which creates a force inducing sliding for the whole filament. After a certain amount of sliding has occurred, the myosin head cannot remain attached, hence the bond is disconnected and the head returns to the original configuration, ready for creating a new bond. At the level of the sarcomere, this mechanism is responsible for bringing Z-disks closer to each other, by reducing the extent of the $\mathrm{H}$-band.

Finally, as in all cells the endoplasmic reticulum is responsible for the transport of proteins. In myocytes, the specific type of reticulum found is called sarcomeric reticulum and is made of closed compartments roughly parallel to the myofibers called longitudinal tubules, which have an essential role in storing and releasing $\mathrm{Ca}^{2+}$ ions. These ions are at the core of electrical activation in the tissue.

\subsection{The sarcomere as a collection of cyclic mechanochemical motors}

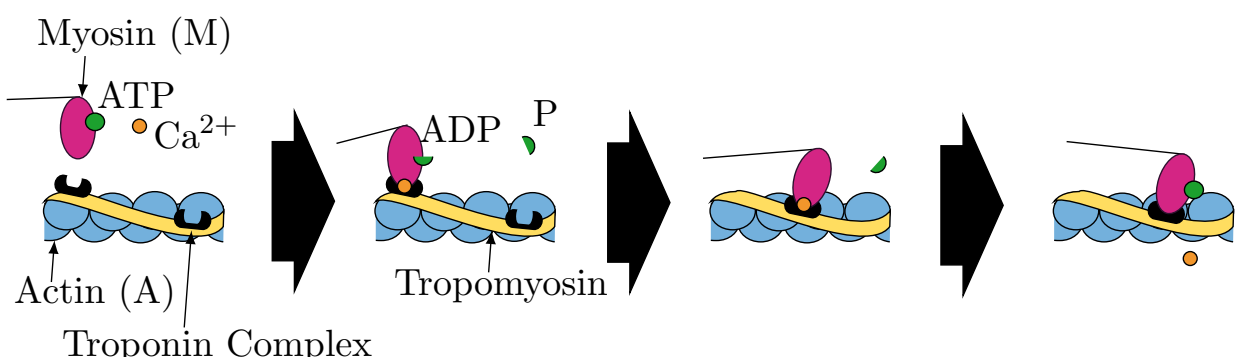

Figure 4: Steps of actin-myosin binding cycle.

We begin with a simplified description of the actin-myosin molecular mechanochemical motors.

The binding of a myosin head to an actin thin filament requires the presence of calcium because the tropomyosin otherwise masks the myosin binding sites on the actin filament. After the cell depolarization, calcium binds to the troponin-C present on actin filaments, thus causing an allosteric change that allows tropomyosin to move, unmasking the myosin binding sites. Myosin with bound ADP (adenosine diphosphate) and P (inorganic phosphate) from a previous ATP (adenosine triphosphate) hydrolysis can then bind to the uncovered binding sites on the actin filament.

Myosin binding on actin is followed by the release of ADP and P, which is coupled to the power stroke, a rotation of the myosin head - from approximately 90 to 45 degrees - that pulls the Z-bands towards each other, thus shortening the sarcomere.

ATP then binds on the attached myosin head, breaking the actin-myosin bond (a lack of ATP would result in the rigid state characteristic of rigor mortis). The myosin head then hydrolyzes the ATP and rotates back to its rest conformation. The complete cycle is depicted in Figure 4.

Myosin binding and unbinding repeat as long as ATP is available and calcium is present on the actin filament. Meanwhile, as part of cell repolarization, calcium is actively pumped back into the sarcoplasmic reticulum. When calcium concentration decreases below $1 \mu \mathrm{mol} . \mathrm{l}^{-1}$, calcium is no longer sufficiently available for the actin filament, the tropomyosin again masks 
the binding sites on the actin filaments, hence contraction ceases until the next action potential wave arises.

Finally, the complete motor cycle corresponding to binding and unbinding can be written in terms of the following chemical and mechanical cycles (with reaction rates indicated over the arrows).

\section{Chemical cycle:}

$$
\begin{gathered}
A M+A T P \stackrel{k_{1}}{\longrightarrow} M . A T P+A \\
M . A T P \stackrel{k_{2}}{\longrightarrow} M . A D P . P \\
M . A D P . P+A \stackrel{k_{3}}{\longrightarrow} A M . A D P . P \\
A M . A D P . P \stackrel{k_{4}}{\longrightarrow} A M+A D P+P
\end{gathered}
$$

This chemical cycling engine is an open thermodynamical system: it is fed with ATP and produces ADP and $\mathrm{P}$.

The first reaction describes the breaking of actin-myosin bridges energized by ATP. It is reversible, leading to a collective behavior of ATP (a single molecule can break several bridges before being hydrolyzed), but for simplicity we average this behavior in a single ATP cycle with a reaction rate $k_{1}$ tuned in consequence, so that the ATP consumption rate is $k_{A T P}=k_{1}[A T P]$.

The concentration of ATP, $[A T P]$, can be considered constant due to the fine control of ATP production by mitochondria and the Krebs cycle, see e.g. [32]. Furthermore, the ATP stock can be neglected compared with higher density energy reserves. This implies a "just-intime ATP production" that leads to the following important property: for these aerobic cardiac cells burning carbohydrates and fatty acids, oxygen consumption is an affine function of ATP consumption (or production). This property - observed at all scales (cell, tissue, organ) - will be used in our future works to couple the mechanics with oxygen feeding through the arterial network (namely, blood perfusion), in particular for the heart.

The second irreversible reaction is the ATP hydrolysis that occurs on unbound myosin heads. At the end of hydrolysis, the binding of myosin heads is again possible as described by the third reaction.

The fourth reaction closes the cycle with the detachment of the products of hydrolysis, $\mathrm{ADP}$ and $\mathrm{P}$. It is in general accompanied by a motion of the freshly bound myosin head towards an equilibrium position. But this motion that produces mechanical energy - called the power stroke - may not occur in the case of a "futile hydrolysis", so that some hypothesis will be done in order to make $k_{3}$ and $k_{4}$ the useful binding and power stroke rates.

For simplicity, $\mathrm{Ca}^{2+}$ ions are not included in these reactions. We will comment further on this point later as calcium has an effect on the unbinding rate.

\section{Mechanical cycle:}

$$
\begin{aligned}
& M_{b} \stackrel{g(\text { ATP })}{\longrightarrow} M_{u} \\
& M_{u} \stackrel{f}{\longrightarrow} M_{b}+W_{m}+A D P+P
\end{aligned}
$$

It describes the evolution of the myosin head through two different states, as summarized in Fig. 4:

- $M_{b}$, the bound head in equilibrium on the actin filament;

- $M_{u}$, the unbound head, out of equilibrium due to ATP hydrolysis; 
- $M_{b}$ again bound in equilibrium after the release of ADP and P. This step produces the mechanical energy $W_{m}$ (power stroke) as a consequence of a forward motion (sliding of the myosin head along the actin filament until it reaches some equilibrium position).

Here $g$ - a function of $[A T P]$ - is the rate of unbinding and ATP hydrolysis, and $f$ is the rate of binding and power stroke.

Tight mechanochemical coupling assumption We suppose that a step along the mechanical cycle is always linked to a step along the chemical cycle. This gives the following correspondence between the reaction rates

$$
\begin{aligned}
g & =k_{1}[A T P], \\
\frac{1}{f} & =\frac{1}{k_{3}[A]}+\frac{1}{k_{4}} .
\end{aligned}
$$

The limiting step in this second two-step reaction starting with binding is the power stroke $\left(k_{4} \ll k_{3}[A]\right)$, so that $f \approx k_{4}$.

Conservation of cycling-myosin hypothesis: We assume an ideal cycle with the same concentration $\left[M_{b}\right]$ at the beginning and at the end of the cycle, so that, in order to have $\dot{M}_{b}=0$, as a consequence of (3) we must have

$$
f=g .
$$

In particular, we have $f=g=k_{A T P}$. In the sequel, we shall consider other sources of myosin head unbinding than ATP binding on the head and $g$ will be larger than $k_{A T P}$.

\subsection{Huxley model of the collective behavior of molecular motors in a sar- comere}

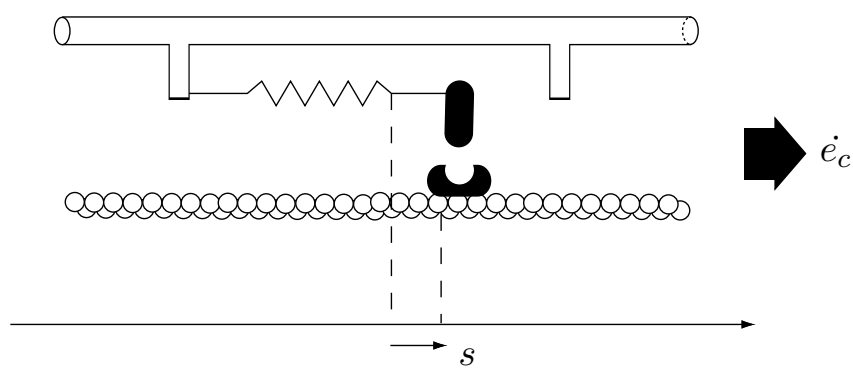

Figure 5: Mechanical modeling scheme of the actin-myosin binding sites

It is possible to translate the chemical cycle (2) into a set of differential equations describing in particular the evolution of the concentration of bridges $[A M]$, but it is not possible to deduce from these equations a force balance equation taking into account the collective behavior of sarcomere motors. For that purpose, Huxley has proposed to use a finer description that we now recall.

We thus follow the main assumptions of [20] where the binding of a myosin head occurs within a certain range $[0, h]$ for the displacement of the tip of the myosin head with respect to a reference position on the actin filament. When dividing the tip displacement by $h$ we then obtain a dimensionless strain quantity $s$, which can be seen as an angular variable modeling the 
fact that the myosin head hinges on its axis like an angular spring, see Figure 5. The Huxley model is a conservation equation for $n(t, s)$, the density of existing bridges with strain $s$ at time $t$, normalized by the total density of actin-myosin pairs, so that $0 \leq n \leq 1$. This model is driven by the mechanochemical mechanisms of creation and destruction of bridges. A rigid actin and myosin filaments hypothesis is made, so that for each active bridge the microscopic strain rate $\dot{s}$ is equal to the macroscopic filament sliding rate $\dot{e}_{c}$. The total derivative of $n$ can then be written $\frac{D n}{D t}=\frac{\partial n}{\partial t}+\dot{e}_{c} \frac{\partial n}{\partial s}$. The binding and unbinding rates $f$ and $g$ and the (microscopic) mechanical energy $W_{m}$ will be now functions of $t$ and $s$. This makes it possible to write a model for the quasi-static equilibrium of the whole collection of myosin molecules considered as springs in parallel being attached to the rigid actin filaments, as follows

$$
\begin{aligned}
& \frac{\partial n}{\partial t}+\dot{e}_{c} \frac{\partial n}{\partial s}=\left(n_{0}-n\right) f-n g \\
& \tau_{c}(t)=\int \frac{\partial W_{m}(t, s)}{\partial s} n(t, s) d s
\end{aligned}
$$

where $n_{0}$ denotes a reduction factor $\left(0 \leq n_{0} \leq 1\right)$ accounting for the fact that all bridges may not be actually recruitable due to external effects which will be discussed later. What is most important at this stage is to emphasize that this additional variable $n_{0}$ - by which we depart from the original Huxley model - may vary in time, but does not depend on $s$.

In this framework, a major step in the modeling effort will consist in determining a good choice of functions $W_{m}, f$ and $g$. To that aim we focus on the model proposed in [3].

\section{Remark 2}

We can link the Huxley density $n$ to the actin-myosin concentration $[A M]$, introducing the total concentrations $\mu_{A}$ and $\mu_{M}$ of actin and myosin, respectively. To that purpose, we use the chemical cycle described in (2). We have

$$
\begin{aligned}
\mu & =\mu_{A}=[A M]+[A]+[A M . A D P . P] \\
& =\mu_{M}=[A M]+[M . A T P]+[A M . A D P . P]+[M . A D P . P],
\end{aligned}
$$

and the Huxley density is linked to $[A M]$ by

$$
\frac{[A M](t)}{\mu}=\int n(t, s) d s .
$$

After some tedious computations, the law of mass action applied to (2) gives the following Michaelis-Menten model (see e.g. Nelson, [28, Chapter 10]):

$$
\frac{d}{d t}\left(\frac{[A M]}{\mu}\right)=k_{A T P}\left(1-\frac{[A M]}{\mu}\right)-k_{A T P} \frac{[A M]}{\mu} .
$$

We recognize in this equation an $s$-averaged version of (4) during a whole cycle with $k_{A T P}$ being the $s$-averaged value of $f$ and $g$.

In order to guide our choice of $W_{m}, f$ and $g$, it is interesting to discuss in what precise sense the sarcomere model (4)-(5) is a motor model.

Motor property and symmetry breaking: A contractile element model has to be able to produce mechanical energy, e.g. to produce a positive stress $\tau_{c}>0$ during contraction $\left(\dot{e}_{c}<0\right)$. In particular, the maximum value of $\tau_{c}$ corresponding to a stalling motor $\left(\dot{e}_{c}=0\right)$ must be positive. This will be our motor property. Maintaining stalling conditions in (4) leads to using the stationary solution of this equation, namely, $n=\frac{f}{f+g}$, so that the motor property is

$$
\int \frac{\partial W_{m}}{\partial s} \frac{f}{f+g} d s>0
$$


Choice of $W_{m}, f$ and $g$ : The simplest choice for $W_{m}$ corresponds to linear spring behavior for the actin-myosin bridges with stiffness $k_{0}$ and possible pre-strain $s_{0} \geq 0$ :

$$
W_{m}(t, s)=\frac{k_{0}}{2}\left(s+s_{0}\right)^{2} .
$$

Even when this potential energy is symmetric $\left(s_{0}=0\right)$ we will break the symmetry (with respect to $s=0)$ of $\frac{\partial W_{m}}{\partial s} \frac{f}{f+g}$ by adequate choices of $f$ and $g$.

In the above analysis we have so far disregarded the effects of calcium concentrations and mechanical factors, indeed. We now take into account the fact that this binding-unbinding cycle occurs only in the presence of calcium ions and with strain values $s \in[0,1]$ to first write

$$
f(t, s)=k_{A T P} \mathbb{1}_{s \in[0,1]} \mathbb{1}_{\left[\mathrm{Ca}^{2+}\right]>C},
$$

where $C$ is a threshold concentration necessary to trigger the initial reaction. The frequency of unbinding is somewhat more complicated because some additional mechanical phenomena must be considered. We know that during the relaxation period $\left[\mathrm{Ca}^{2+}\right]<C$ and bridges are destructed with the intake of the calcium ions by the sarcoplasmic reticulum pumps with a kinetic constant $k_{R S}$ (different from $k_{A T P}$ ). But we also have destruction of some bridges when the relative speed between myosin and actin is too high from a mechanical standpoint during both relaxation and contraction. Finally, the complete function $g$ can be modeled by

$$
g(t, s)=\alpha\left|\dot{e}_{c}\right|+k_{A T P} \mathbb{1}_{s \notin[0,1]} \mathbb{1}_{\left[\mathrm{Ca}^{2+}\right]>C}+k_{R S} \mathbb{1}_{\left[\mathrm{Ca}^{2+}\right]<C} .
$$

Remark that, when $\left[\mathrm{Ca}^{2+}\right]>C, \frac{\partial W_{m}}{\partial s} \frac{f}{f+g} \geq 0$ and $>0$ on $] 0,1[$, so that the motor property holds.

It is then possible to summarize the effect of calcium concentration - entering in the two functions $f$ and $g$ with indicator functions of disjoint supports - with only one control variable denoted by $u$ and defined by

$$
\begin{gathered}
u(t)=|u(t)|_{+}-|u(t)|_{-} \text {with }\left\{\begin{array}{l}
|u(t)|_{+}=k_{A T P} \mathbb{1}_{\left[\mathrm{Ca}^{2+}\right]>C} \\
|u(t)|_{-}=k_{R S} \mathbb{1}_{\left[\mathrm{Ca}^{2+}\right]<C}
\end{array}\right. \\
f(s, t)=|u(t)|_{+} \mathbb{1}_{s \in[0,1]}, \\
g(s, t)=|u(t)|+\alpha\left|\dot{e}_{c}\right|-f(s, t) .
\end{gathered}
$$

This control variable $u$ is a function of the ions concentration only, and derives directly from the electrical activation model - after adequately identifying $k_{A T P}$ and $k_{R S}$.

\subsection{Moments equations}

In order to obtain a constitutive law applicable in the context of continuum mechanics, we need to translate the previous microscopic description to a mesoscopic scale. To that aim, we will compute from $n$ some quantities averaged on the whole population of bridges. Hence, we define the moments

$$
\mu_{p}=\int_{\mathbb{R}} s^{p} n(s, t) d s .
$$

We then point out that the judicious choice of the functions $f$ and $g$-in particular such that $f+g$ is independent of $s$-allows to obtain the following recursive relation defining a $p$ th-order moment with only the previous order moment,

$$
\begin{aligned}
\dot{\mu}_{p} & =\int_{\mathbb{R}} s^{p}\left(n_{0} f-n(f+g)-\dot{e}_{c} \frac{\partial n}{\partial s}\right) d s \\
& =n_{0} f_{p}-(f+g) \mu_{p}+p \dot{e}_{c} \mu_{p-1},
\end{aligned}
$$


where $f_{p}=\int_{\mathbb{R}} s^{p} f d s$ is the $p$ th-order moment of $f$.

Remark that - for more general choices of $f$ and $g$ with $f+g$ dependent on $s$ - the moment $\mu_{p}$ would be in essence coupled to moments at all other orders by the resulting dynamical equation. Some closure relations could then be used to limit moment equations to a finite dimensional system. These relations are usually some "laws" linking e.g. two moments in a manner consistent with experiments. We will come back later to the closure relations hidden behind our choice of $f$ and $g$.

Considering our choice for $W_{m}$, the first two moments are well-adapted to the definition of natural macroscopic variables considering the sarcomere as a collection of statistical springs:

1. 0th-order moment: with an appropriate rescaling, this represents the equivalent stiffness of the sarcomere in the current configuration of active bridges, which we denote by $k_{c}$

$$
k_{c}=k_{0} \int_{\mathbb{R}} n(s, t) d s .
$$

2. 1st-order moment: let $\tau_{c}$ be the stress associated with the set of "springs" considered at equilibrium when $s=-s_{0} \leq 0$ - meaning that for $s=0$ the spring is under tension:

$$
\tau_{c}=k_{0} \int_{\mathbb{R}}\left(s+s_{0}\right) n(s, t) d s .
$$

Therefore, the differential equation system verified by the first two moments leads to a constitutive law - close to that derived in [4] - summarized in

$$
\left\{\begin{array}{l}
\dot{k}_{c}=-\left(|u|+\alpha\left|\dot{e}_{c}\right|\right) k_{c}+n_{0} k_{0}|u|_{+} \\
\dot{\tau}_{c}=-\left(|u|+\alpha\left|\dot{e}_{c}\right|\right) \tau_{c}+\dot{e}_{c} k_{c}+n_{0} \sigma_{0}|u|_{+}
\end{array}\right.
$$

where we have defined $\sigma_{0}=\left(\frac{1}{2}+s_{0}\right) k_{0}$. We can see that e.g. the stationary behaviors of this first-order dynamical system lead to

$$
\begin{cases}k_{c}=n_{0} k_{0}, \quad \tau_{c}=n_{0} \sigma_{0}, & \text { when } u>0 \text { (contraction) } \\ k_{c}=0, \quad \tau_{c}=0, & \text { when } u \leq 0 \text { (relaxation) }\end{cases}
$$

assuming that $n_{0}$ is constant (or slowly-varying) in time, and this also shows the direct effect of $n_{0}$ on the contraction behavior.

To this stress $\tau_{c}$, we add one contribution to account for the fact that the binding-unbinding phenomenon dissipates energy by friction and viscous effects, which gives

$$
\sigma_{c}=\tau_{c}+\mu_{c} \dot{e}_{c}
$$

We now comment on our choices of $f$ and $g$ in terms of closure relations associated with observed behaviors of sarcomeres. We thus give three properties of the model (9), namely: the Mirsky-Parmley relation for passive relaxation [25] ; the Hill relation for isotonic contraction [15], and rate-independence of the sarcomere stress-strain cycles.

Mirsky-Parmley relation for passive relaxation: During diastole the cardiac tissues are relaxing in order to allow filling of the heart. Passive relaxation (without active calcium pumping) corresponds to $u=0$. The solution of (9) is then given by

$$
k_{c}(t)=k_{c}\left(t_{0}\right) \exp \alpha\left(\sigma\left(t_{0}\right)-\sigma(t)\right), \text { with } \sigma(t)=\int_{0}^{t}\left|\dot{e}_{c}(\theta)\right| d \theta \text {, and } \frac{d \tau_{c}}{d \sigma}=-\alpha \tau_{c}+k_{c},
$$

which is exactly the Mirsky-Parmey model. When $k_{c}$ is supposed constant, integrating the last equation leads also to a standard visco-plastic constitutive law (cf. [11]). 
Hill relation for isotonic sarcomere contraction: This is a particular case of contraction where the maximum shortening velocity $\left(\dot{e}_{c}<0\right)$ is measured during a contraction $(u>0)$ with $\tau_{c}$ constant. Using $\dot{\tau}_{c}=0$ in (9) leads to

$\dot{e}_{c}(t)=\frac{\tau_{c}-n_{0} \sigma_{0}}{\alpha \tau_{c}+k_{c}(t)} u$, with $k_{c}$ solution of $\dot{k}_{c}=-\frac{u}{\alpha \tau_{c}+k_{c}}\left[k_{c}^{2}-k_{c}\left(n_{0} k_{0}-\alpha n_{0} \sigma_{0}\right)-\alpha n_{0} k_{0} \tau_{c}\right]$,

hence, the homographic relation between $\dot{e}_{c}$ and the load $\tau_{c}$ (Hill's law) is satisfied. Remark that the constitutive law proposed by Hunter et al [19] is in itself a direct adaptation of Hill's law.

Rate-independence of the sarcomere stress-strain cycles: This last property describes the dependence of the solution of (9) upon the frequency in the case of periodic inputs, a situation of importance for the heart. In fact, when $u$ is chosen as a rate function (e.g. ATP or $\mathrm{Ca}^{2+}$ flow rates), it is easy to check that (9) is invariant under changes of the time scale. In particular, this means that any closed $\tau_{c}-e_{c}$ cycle will be independent of the frequency in a periodic regime. This is a property observed for a large range of frequencies from the present cell scale to the organ scale (pressure-volume cycle). It is important because the cardiac output is then mainly under the control of the heart rate, the stroke volume being quite insensitive to the frequency.

Remark that this counter-intuitive property is intrinsic to the contractile element behavior on the cell scale but it is hindered by added viscous damping. Some control mechanisms - not studied here - tend to preserve the property despite damping: it will be the case of calcium dynamics in the cell (positive staircase effect) or of the sympathetic action of the autonomous nervous system for the whole organ.

This property is also key from a mathematical viewpoint: $e_{c} \rightarrow \tau_{c}$ is a rate independent hysteresis operator which has some nice regularity properties. This has been used in [21] to study the well-posedness of the equations of motion of a simplified 1D heart model associated with the constitutive law (9).

\section{Remark 3}

Observe that since we always have $|u|_{+} \leq|u|$ (and recalling that $0 \leq n_{0} \leq 1$ ) the differential equation satisfied by the stiffness $k_{c}$ guarantees the bounds

$$
0 \leq k_{c} \leq k_{0}
$$

provided that $k_{c}$ is initialized in this interval.

\section{Remark 4}

The reduction factor $n_{0}$ allows to take into account the Starling effect by which the maximum value that can be reached by the active stress depends on the strain $e_{c}$. In this case, $n_{0}$ can be defined as a function of the history of $e_{c}$, e.g. to represent the effect of the previous maximum stretch (or pre-load in cardiac behavior) - usually considered to be a positive effect when this value increases in a reasonable range - or even some longer-term effects spanning over several loading cycles (or heart beats). Note that $n_{0}$ could then be modeled by a dynamical system with $e_{c}$ as an input. Likewise, $n_{0}$ could be used to adjust the coupling of this tissue model to a perfusion model accounting for the oxygen supply - via the energy flow, see also Remark 5 below. 


\subsection{Energetic balance in the sarcomere}

Once more, the moments - the second-order moment here - provide a meaningful mesoscopic variable. Indeed, the elastic energy stored in one microscopic spring is $W_{m}(s, t)=\frac{k_{0}}{2}\left(s+s_{0}\right)^{2}$, so we can introduce the energy averaged over the collection of bridges

$$
U_{c}=\frac{k_{0}}{2} \int_{\mathbb{R}}\left(s+s_{0}\right)^{2} n(s, t) d s .
$$

Using the moments equation (8) we get

$$
\begin{aligned}
\dot{U}_{c} & =\frac{k_{0}}{2} \int_{\mathbb{R}}\left(s+s_{0}\right)^{2} f d s-\left(|u|+\alpha\left|\dot{e}_{c}\right|\right) U_{c}+\dot{e}_{c} \tau_{c} \\
& =-\left(|u|+\alpha\left|\dot{e}_{c}\right|\right) U_{c}+\dot{e}_{c} \tau_{c}+n_{0} U_{0}|u|_{+},
\end{aligned}
$$

with $U_{0}=\frac{k_{0}}{2}\left(s_{0}^{2}+s_{0}+\frac{1}{3}\right)>0$.

\section{Remark 5}

Remark that the incoming energy flow rate $n_{0} U_{0}|u|_{+}=\lambda n_{0} k_{0} \mathbb{1}_{\left[\mathrm{Ca}^{2+}\right]>C}$ for some positive parameter $\lambda$. In this form, the main energy control inputs are apparent. Recall first that in the aerobic heart oxidization of substrates (mainly free fatty acids) at the origin of ATP production is directly dependent upon the workload (here the sum of the power of external forces and of the dissipative terms). This workload has to be seen as the energy demand of the heart to ensure its cardiac output (the main function). This energy demand is the input of regulation mechanisms of fatty acid oxidization, hence of ATP production. We have not described these (not well known) mechanisms here (see e.g. [1]). Our model is "open loop" with the following possible inputs:

- $k_{0} n_{0}$ is the "maximal contractility input" (dependent upon the size $n_{0}$ of the actin-myosin pairs recruitable in motors. It is under the control of the heart set point, in particular the filling (Starling effect);

- $\mathbb{1}_{\left[\mathrm{Ca}^{2+}\right]>C}$ is the calcium input having a global chronotropic effect trough the heart rate and a local inotropic effect through the duty ratio of this $0 / 1$ signal. This is highly dependent upon the calcium dynamics.

We point out that this energy demand is in direct correspondence with the oxygen demand of the heart (due to the precise control of ATP levels) and that the oxygen demand / supply ratio is also under control in the coronary bed.

While the energy variable $U_{c}$ has a clear physical meaning at the microscopic scale, we also wish to define an energy directly from the mesoscopic variables, because this quantity will then be useful to prove existence results or discretize - especially in time - the system. In this respect, the natural energy that can be defined from $\tau_{c}$ and $k_{c}$ is the following elastic energy

$$
\Psi_{c}=\frac{1}{2 k_{c}} \tau_{c}^{2},
$$

for which we have the dynamics

$$
\begin{aligned}
\dot{\Psi}_{c} & =\frac{\tau_{c}}{k_{c}} \dot{\tau}_{c}-\frac{1}{2 k_{c}} \tau_{c}^{2} \frac{\dot{k}_{c}}{k_{c}} \\
& =-\left(|u|+\alpha\left|\dot{e}_{c}\right|\right) \Psi_{c}+\dot{e}_{c} \tau_{c}+n_{0} k_{0}\left(\frac{\tau_{c}}{k_{c}}\left(s_{0}+\frac{1}{2}-\frac{1}{2} \frac{\tau_{c}}{k_{c}}\right)\right)|u|_{+} .
\end{aligned}
$$


We then observe that the local elastic energy $\Psi_{c}=\frac{\tau_{c}^{2}}{2 k_{c}}$ is always bounded by the stored energy $U_{c}$. Indeed, noticing that $U_{0} \geq \frac{\sigma_{0}^{2}}{2 k_{0}}=\frac{k_{0}}{2}\left(\frac{1}{2}+s_{0}\right)^{2}$ we have

$$
\begin{aligned}
\frac{d}{d t}\left(U_{c}-\Psi_{c}\right) & \geq-\left(|u|+\alpha|\dot{e}|_{c}\right)\left(U_{c}-\Psi_{c}\right)+\left(\left(\frac{1}{2}+s_{0}\right)^{2}-2\left(\frac{\tau_{c}}{k_{c}}\right)\left(\frac{1}{2}+s_{0}\right)+\left(\frac{\tau_{c}}{k_{c}}\right)^{2}\right) \frac{n_{0} k_{0}}{2}|u|_{+} \\
& \geq-\left(|u|+\alpha|\dot{e}|_{c}\right)\left(U_{c}-\Psi_{c}\right)+\left(\left(\frac{\tau_{c}}{k_{c}}\right)-\left(\frac{1}{2}+s_{0}\right)\right)^{2} n_{0} k_{0}|u|_{+} \\
& \geq-\left(|u|+\alpha|\dot{e}|_{c}\right)\left(U_{c}-\Psi_{c}\right) .
\end{aligned}
$$

Using the Gronwall theorem, this implies that

$$
U_{c} \geq \Psi_{c}
$$

holds at all times since it is satisfied at the end of a contraction phase $(u>0)$ where we have the asymptotic behavior

$$
\dot{e}_{c} \rightarrow 0, \quad k_{c} \rightarrow n_{0} k_{0}, \quad \tau_{c} \rightarrow n_{0} \sigma_{0}, \quad U_{c} \rightarrow n_{0} U_{0} .
$$

\section{Energy-preserving formulation of the active tissue model}

\subsection{Rheological model}

Complex non-homogenous mechanical behaviors can be represented by rheological models, see in particular [22], and this holds in particular in the context of biological tissues [15]. The main idea is to describe the global macroscopic behavior resulting from the combination of several constitutive elements by an analogy with a combination of spring and damping elements in series and parallel. Under the small displacements assumption, the natural rules of series and parallel associations apply. However, when considering the general non-linear case - with large displacements and deformations - the combined laws are more intricate as we now explain.

Let us consider a 1D element corresponding to two elastic materials in series. This rheological component will be used to represent the tissue behavior along the fibre direction, which is why the one-dimensional setting is adequate. We then have for the deformation maps

$$
\phi=\phi_{2} \circ \phi_{1} \Rightarrow \nabla \phi=\nabla \phi_{2} \cdot \nabla \phi_{1},
$$

so that the corresponding Green-Lagrange strains compose as

$$
1+2 e=\left(1+2 e_{1}\right)\left(1+2 e_{2}\right) .
$$

Considering the stresses, we sum the free energies $W(e)=W_{1}\left(e_{1}\right)+W_{2}\left(e_{2}\right)$ under the kinematical constraint (14). Minimizing the energy allows then to characterize $e_{1}$ and $e_{2}$ at the equilibrium

$$
\left(e_{1}, e_{2}\right)=\underset{1+2 e=\left(1+2 e_{1}\right)\left(1+2 e_{2}\right)}{\operatorname{argmin}} W(e),
$$

so that we have the stationarity conditions

$$
\left\{\begin{array}{l}
W_{1}^{\prime} \mathrm{d} e_{1}+W_{2}^{\prime} \mathrm{d} e_{2}=0 \\
\left(1+2 e_{2}\right) \mathrm{d} e_{1}+\left(1+2 e_{1}\right) \mathrm{d} e_{2}=0
\end{array}\right.
$$

with $W_{1}^{\prime}$ and $W_{2}^{\prime}$ the derivatives of $W_{1}$ and $W_{2}$ with respect to the scalar strains $e_{1}$ and $e_{2}$, respectively. We then obtain

$$
\frac{W_{1}^{\prime}}{1+2 e_{2}}=\frac{W_{2}^{\prime}}{1+2 e_{1}} \text {. }
$$


Finally, the total stress in the element $\sigma=\frac{\partial W}{\partial e}$ is thus given by

$$
\begin{aligned}
\mathrm{d} W & =\sigma \mathrm{d} e=W_{1}^{\prime} \mathrm{d} e_{1}+W_{2}^{\prime} \mathrm{d} e_{2} \\
& =\frac{W_{1}^{\prime}}{1+2 e_{2}}\left(\left(1+2 e_{2}\right) \mathrm{d} e_{1}+\left(1+2 e_{1}\right) \mathrm{d} e_{2}\right) \\
& =\frac{W_{1}^{\prime}}{1+2 e_{2}} \mathrm{~d} e
\end{aligned}
$$

and similarly when switching the index 1 and 2. Therefore, the series rheological law finally gives

$$
\sigma=\frac{\sigma_{1}}{1+2 e_{2}}=\frac{\sigma_{2}}{1+2 e_{1}} .
$$

\subsection{Active tissue rheological assembly}

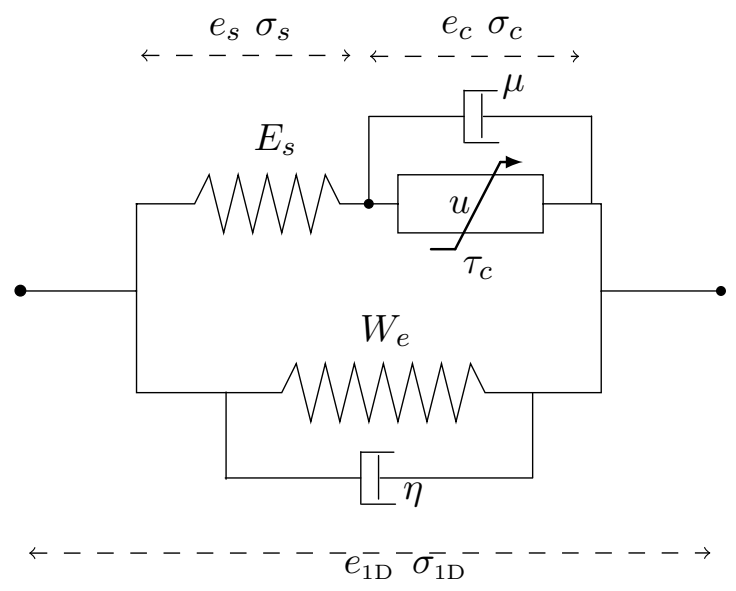

Figure 6: Complete rheological model.

We can now proceed to the final assembly of passive and active elements using non-linear rheological schemes as presented in [7]. The final scheme is described in Figure 6 where we see:

- The active component of the sarcomere described by the above contraction model

$$
\sigma_{c}=\tau_{c}+\mu_{c} \dot{e}_{c}
$$

- In series, an elastic element describing the $Z$ disks. This element plays an important role in the isometric behavior of the cardiac fiber, since the active element can then contract $\left(e_{c}<0\right)$ without any deformation of the entire fiber. Denoting by $\left(e_{s}, \sigma_{s}\right)$ the one-dimensional strain and stress of this element, we assume

$$
\sigma_{s}=E_{s} e_{s}
$$

and the rheological model discussed in Section 4.1 implies

$$
\sigma_{c}=\tau_{c}+\mu \dot{e}_{c}=E_{s} \frac{e_{1 \mathrm{D}}-e_{c}}{\left(1+2 e_{c}\right)^{3}}\left(1+2 e_{1 \mathrm{D}}\right),
$$

where $e_{1 \mathrm{D}}=\underline{\tau}_{1} \cdot \underline{e} \cdot \underline{\tau}_{1}$ is the strain along the fiber direction characterized by the unit vector $\underline{\tau}_{1}$, and we have according to the above series kinematics

$$
1+2 e_{1 \mathrm{D}}=\left(1+2 e_{s}\right)\left(1+2 e_{c}\right) .
$$


- In parallel, the additional element represents the collagen and elastin matrix surrounding the fiber. This passive element is fully $3 \mathrm{D}$ and is assumed to be described by

$$
\underline{\underline{\Sigma}}_{p}=\frac{\partial W_{e}}{\partial \underline{\underline{e}}}(\underline{\underline{e}})+\frac{\partial W_{v}}{\partial \underline{\underline{\dot{e}}}}(\underline{\underline{e}}, \underline{\underline{e}})
$$

where $W_{e}$ denotes a hyperelastic potential, and $W_{v}$ a viscous pseudo-potential assumed to satisfy the dissipation property

$$
\frac{\partial W_{v}}{\partial \underline{\underline{\dot{e}}}}(\underline{\underline{e}}, \underline{\underline{\dot{e}}}): \underline{\underline{\dot{e}}} \geq 0, \quad \forall(\underline{\underline{e}}, \underline{\underline{\dot{e}}}) .
$$

For example we can use an isotropic constitutive law in the parallel branch, in which case the hyperelastic potential is necessarily a function of the so-called reduced invariants $\left(J_{1}, J_{2}, J\right)$ of the tensor $\underline{\underline{C}}$, according to the Rivlin-Ericksen theorem [30]. In particular, it is classical to consider combinations of polynomials in the terms $\left(J_{1}-3\right)$ (neo-Hookean) and $\left(J_{2}-3\right)$ (Mooney-Rivlin like), with an additional term penalizing compressible behaviors such as $(J-1-\ln J)$ weighed by a large coefficient [9,23], and sometimes also exponential terms (also applied on similar polynomials) to represent specific stiffening effects of living tissues [33]. Note that - even with such an isotropic passive part - the overall behavior would then be non-isotropic - here transversely isotropic - due to the series element contribution exerted along the muscle fiber direction. Nevertheless, we can also - of course - consider anisotropic stress-strain laws in the parallel element, incorporating at least one additional invariant representing a privileged direction in the tissue - for example associated with collagen fibers - defined by

$$
J_{4}=\left(\underline{\tau}_{2} \cdot \underline{\underline{e}} \cdot \underline{\tau}_{2}\right) J^{-\frac{1}{3}},
$$

where $\underline{\tau}_{2}$ denotes a unit vector associated with this direction, and likewise this invariant can be used in polynomial and exponential terms [17, 10,2]. See also [29] for an extension of the Rivlin-Ericksen theorem in the anisotropic case. As concerns the viscous pseudopotential $W_{v}$, the positiveness property (19) holds in particular when $W_{v}$ is a convex function of $\underline{\underline{\dot{e}}}$ with $\frac{\partial W_{v}}{\partial \underline{\underline{e}}}(\underline{\underline{e}}, \underline{\underline{0}})=\underline{\underline{0}}$, as for the simple extension of Rayleigh damping given by $W_{v}=\frac{\eta}{2} \operatorname{tr}(\underline{\underline{\dot{e}}})^{2}$.

The whole assembly of these elements defines the total stress tensor

$$
\underline{\underline{\Sigma}}=\underline{\underline{\Sigma}}_{p}+\sigma_{1 \mathrm{D}} \underline{\tau}_{1} \otimes \underline{\tau}_{1},
$$

with the specific stress for the sarcomere, recall (15),

$$
\sigma_{1 \mathrm{D}}=\frac{\sigma_{c}}{1+2 e_{s}}=\frac{\sigma_{s}}{1+2 e_{c}} .
$$




\subsection{Energy-preserving variational formulation}

By combining the fundamental law of dynamics and the above constitutive laws, the overall formulation of our problem can be summarized as follows

$$
\left\{\begin{array}{l}
\underline{\dot{y}}=\underline{v} \\
\int_{\Omega_{0}} \rho \underline{\underline{v}} \cdot \underline{v}^{*} d \Omega+\int_{\Omega_{0}} \underline{\underline{\Sigma}}: \mathrm{d}_{\underline{y}} \underline{\underline{e}} \cdot \underline{v}^{*} d \Omega=\mathcal{P}_{\text {ext }}\left(\underline{v}^{*}\right), \quad \forall \underline{v}^{*} \in V \\
\underline{\underline{\Sigma}}=\frac{\partial W_{e}}{\partial \underline{\underline{e}}}+\frac{\partial W_{v}}{\partial \underline{\underline{\dot{e}}}}+\sigma_{1 \mathrm{D}} \underline{\tau}_{1} \otimes \underline{\tau}_{1} \\
\left(\tau_{c}+\mu \dot{e}_{c}\right)\left(1+2 e_{c}\right)^{3}=E_{s}\left(e_{1 \mathrm{D}}-e_{c}\right)\left(1+2 e_{1 \mathrm{D}}\right) \\
\dot{k}_{c}=-\left(|u|+\alpha\left|\dot{e}_{c}\right|\right) k_{c}+n_{0} k_{0}|u|_{+} \\
\dot{\tau}_{c}=-\left(|u|+\alpha\left|\dot{e}_{c}\right|\right) \tau_{c}+\dot{e}_{c} k_{c}+n_{0} \sigma_{0}|u|_{+}
\end{array}\right.
$$

under the above definition of the fiber stress and strain

$$
\sigma_{1 \mathrm{D}}=\left(\frac{1+2 e_{c}}{1+2 e_{1 \mathrm{D}}}\right) \sigma_{c}, \quad \sigma_{c}=\tau_{c}+\mu \dot{e}_{c} \text { and } e_{1 \mathrm{D}}=\underline{\tau}_{1} \cdot \underline{\underline{e}} \cdot \underline{\tau}_{1},
$$

and where $\mathcal{P}_{\text {ext }}\left(\underline{v}^{*}\right)$ is the virtual work associated with the external forces. For detailed validations of this model used to represent the heart behavior, see in particular [31, 8, 6].

In order to obtain a mechanical energy relation we use the rheological laws leading to the identity

$$
\underline{\underline{\Sigma}}: \mathrm{d}_{\underline{y}} \underline{\underline{e}} \cdot \underline{v}^{*}=\underline{\Sigma}_{p}: \mathrm{d}_{\underline{y}} \underline{\underline{e}} \cdot \underline{v}^{*}+\sigma_{s} \mathrm{~d} e_{s} \cdot \underline{v}^{*}+\sigma_{c} \mathrm{~d} e_{c} \cdot \underline{v}^{*} .
$$

Hence, when choosing the actual velocity as a test function we get

$$
\underline{\underline{\underline{\Sigma}}}: \underline{\underline{\dot{e}}}=\underline{\underline{\underline{\Sigma}}}: \underline{\underline{\dot{e}}}+\sigma_{s} \dot{e}_{s}+\sigma_{c} \dot{e}_{c}
$$

which gives the total energy balance

$$
\begin{aligned}
& \frac{d}{d t}\left(\mathcal{K}+\mathcal{E}_{e}+\frac{1}{2} \int_{\Omega_{0}} E_{s} e_{s}^{2} d \Omega+\int_{\Omega_{0}} U_{c} d \Omega\right)= \\
& \mathcal{P}_{\text {ext }}(v)+\int_{\Omega_{0}} n_{0} U_{0}|u|_{+} d \Omega-\int_{\Omega_{0}}\left(|u|+\alpha\left|\dot{e}_{c}\right|\right) U_{c} d \Omega-\int_{\Omega_{0}} \mu\left(\dot{e}_{c}\right)^{2} d \Omega-\int_{\Omega_{0}} \frac{\partial W_{v}}{\partial \underline{\underline{\dot{e}}}}: \underline{\underline{\dot{e}}} d \Omega .
\end{aligned}
$$

In this identity, the various energy contributions are as follows:

- $\mathcal{K}=\frac{1}{2} \int_{\Omega_{0}} \rho|\underline{v}|^{2} d \Omega$ is the kinetic energy;

- $\mathcal{E}_{e}=\int_{\Omega_{0}} W_{e} d \Omega$ is the hyperelastic energy of the $3 \mathrm{D}$ matrix;

- $\frac{1}{2} \int_{\Omega_{0}} E_{s} e_{s}^{2} d \Omega$ is an additive passive elastic energy associated with the fiber;

- $U_{c}$ is the microscopic elastic energy of the actin-myosin bridges.

For the source terms:

- $\mathcal{P}_{\text {ext }}$ is the power of external forces;

- $\int_{\Omega_{0}} n_{0} U_{0}|u|_{+} d \Omega$ is the positive power consumed by the actin-myosin engine. 
As for the dissipative terms:

- $\int_{\Omega_{0}}\left(|u|+\alpha\left|\dot{e}_{c}\right|\right) U_{c} d \Omega$ is the energy dissipated during the binding-unbinding mechanisms;

- $\int_{\Omega_{0}} \mu\left(\dot{e}_{c}\right)^{2} d \Omega$ is the (passive) viscous dissipation in the contractile element;

- $\int_{\Omega_{0}} \frac{\partial W_{v}}{\partial \underline{\underline{\underline{e}}}}: \underline{\underline{\dot{e}}} d \Omega$ is the dissipated energy due to the passive viscosity of the $3 \mathrm{D}$ matrix.

These dissipative terms correspond to the entropy production terms in the second law of thermodynamics.

This energy balance is - of course - extremely important from a physical point of view. Note that we have written the balance in a general and exact form without presuming on the relative weights of the various terms in a specific applicative context. In particular, in actual muscular tissues the importance of inertia forces - hence, of kinetic energy - is a matter of debate and needs to be carefully assessed, which should be performed in the light of the balance. It is also very valuable from a mathematical standpoint, since it provides an a priori bound on the solutions of the model - including for the active stress $\tau_{c}$ recalling the inequality $U_{c} \geq \Psi_{c}$ - which is an essential prerequisite to establish existence and uniqueness results, as well as error estimates. This type of mathematical analysis can be performed at least in a linearized framework, see [21].

\section{Discretization of the formulation}

\subsection{A first-order time discretization scheme}

The scheme we propose here is a combination of

- a mid-point energy-conserving extension of the mid-point Newmark scheme for the main mechanical equations, namely, with a specific treatment of the passive stress tensor according to [13] (see also $[24,14]$ ) and an adequate discretization of the series law to preserve the energy balance;

- a first-order monotone scheme for the active law to guarantee the positiveness of the Huxley variables $k_{c}$ and $U_{c}$ and to preserve the upper bound on the local elastic energy $\Psi_{c}$.

In the sequel we use the standard notation

$$
g^{n+\frac{1}{2}}=\frac{g^{n}+g^{n+1}}{2},
$$

except when specified otherwise for some variables that we denote by $g^{n+\frac{1}{2} \sharp}$.

The resulting numerical scheme then reads: 
For $0 \leq n \leq N$, with $\underline{y}^{n}, \underline{v}^{n},\left(\frac{\tau_{c}}{\sqrt{k_{c}}}\right)^{n}, k_{c}^{n}, e_{c}^{n}$ known, compute $\underline{y}^{n+1}, \underline{v}^{n+1},\left(\frac{\tau_{c}}{\sqrt{k_{c}}}\right)^{n+1}, k_{c}^{n+1}, e_{c}^{n+1}$ solutions of the following system

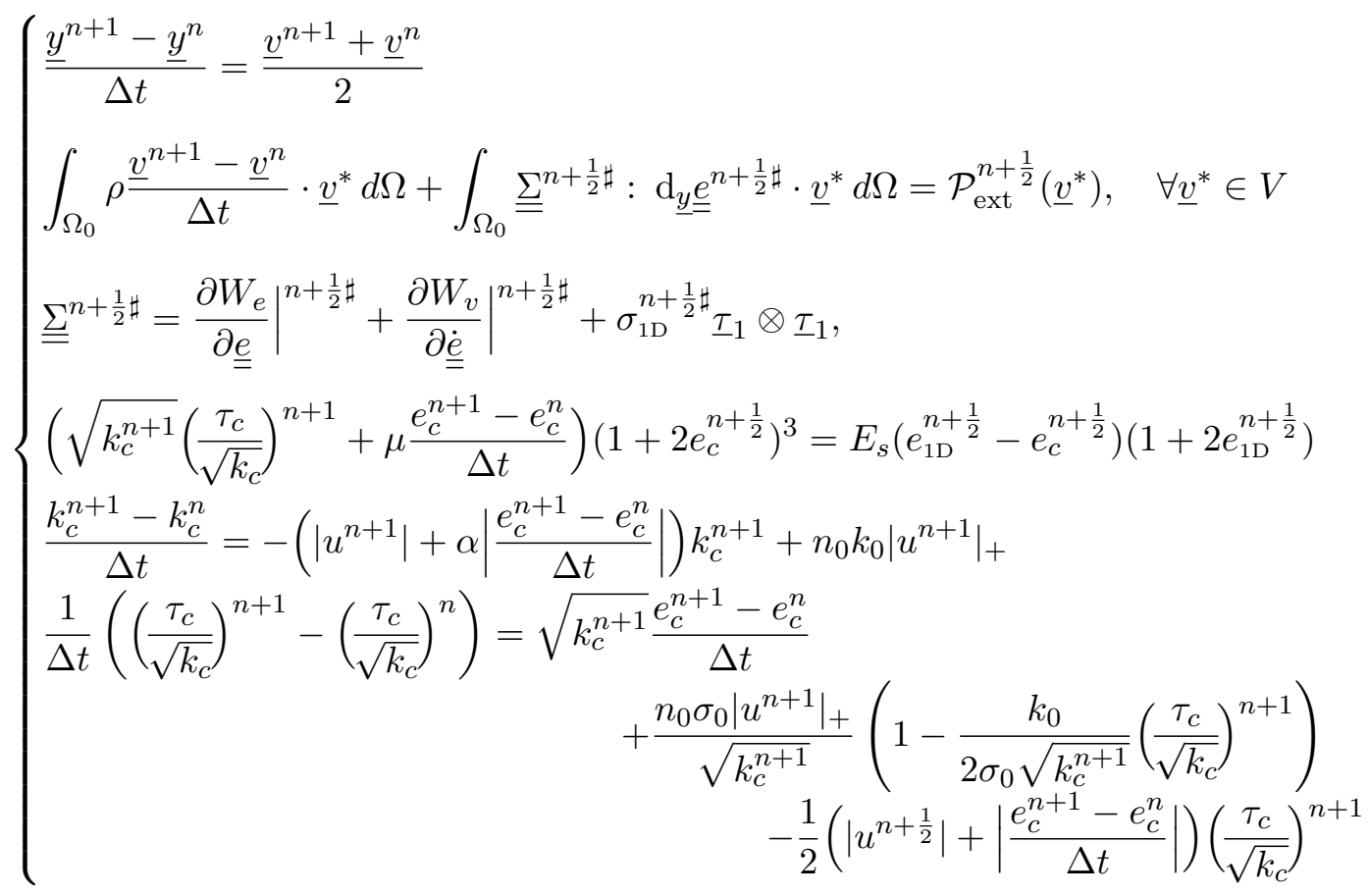

The non-standard mid-point variables have a somewhat classical definition for the strain values

$$
\begin{gathered}
\underline{\underline{e}}^{n+\frac{1}{2} \sharp}=\underline{\underline{e}}^{\left(\underline{y}^{n+\frac{1}{2}}\right),} \\
\left.\mathrm{d}_{\underline{y}} \underline{e}^{n+\frac{1}{2} \sharp} \cdot \underline{v}^{*}=\frac{1}{2}\left(\underline{\underline{F}} \underline{\underline{y}}^{n+\frac{1}{2}}\right)^{T} \cdot \underline{\underline{\nabla}}_{\underline{\xi}} \underline{v}^{*}+\underline{\nabla}_{\underline{\xi}}^{T} \underline{v}^{*} \cdot \underline{\underline{F}}\left(\underline{y}^{n+\frac{1}{2}}\right)\right), \\
\underline{\underline{e}}^{n+\frac{1}{2} \sharp}=\frac{\underline{\underline{e}}^{n+1}-\underline{\underline{e}}^{n}}{\Delta t},
\end{gathered}
$$

but include as in $([13,24,14])$ an energy correction term for the passive elastic stress

$$
\begin{aligned}
\left.\frac{\partial W_{e}}{\partial \underline{\underline{e}}}\right|^{n+\frac{1}{2} \sharp}=\frac{\partial W_{e}}{\partial \underline{\underline{e}}}\left(\underline{\underline{e}}^{n+\frac{1}{2} \sharp}\right) & \\
& +\left(\frac{W_{e}\left(\underline{\underline{e}}^{n+1}\right)-W_{e}\left(\underline{\underline{e}}^{n}\right)}{\Delta t}-\frac{\partial W_{e}}{\partial \underline{\underline{e}}}\left(\underline{\underline{e}}^{n+\frac{1}{2} \sharp}\right): \underline{\underline{e}}^{n+\frac{1}{2} \sharp}\right) \frac{\underline{\underline{e}}^{n+\frac{1}{2} \sharp}}{\underline{\underline{e}}^{n+\frac{1}{2} \sharp}: \underline{\underline{e}}^{n+\frac{1}{2} \sharp}},
\end{aligned}
$$

while viscous stresses are given by

$$
\left.\frac{\partial W_{v}}{\partial \underline{\underline{\dot{e}}}}\right|^{n+\frac{1}{2} \sharp}=\frac{\partial W_{v}}{\partial \underline{\underline{\dot{e}}}}\left(\underline{\underline{e}}^{n+\frac{1}{2} \sharp}\right) .
$$

The series fiber stress is given by the combined formula

$$
\sigma_{1 \mathrm{D}}^{n+\frac{1}{2} \sharp}=\frac{\sigma_{s}^{n+\frac{1}{2} \sharp}}{1+2 e_{c}^{n+\frac{1}{2}}}=\frac{\sigma_{c}^{n+\frac{1}{2} \sharp}}{1+2 e_{s}^{n+\frac{1}{2}}},
$$

with

$$
\sigma_{s}^{n+\frac{1}{2} \sharp}=E_{s} e_{s}^{n+\frac{1}{2}}
$$


and

$$
\sigma_{c}^{n+\frac{1}{2} \sharp}=\sqrt{k_{c}^{n+1}}\left(\frac{\tau_{c}}{\sqrt{k_{c}}}\right)^{n+1}+\mu \frac{e_{c}^{n+1}-e_{c}^{n}}{\Delta t} .
$$

To all these definitions, we add for completeness the auxiliary energy equation

$$
\frac{U_{c}^{n+1}-U_{c}^{n}}{\Delta t}=\sqrt{k_{c}^{n+1}}\left(\frac{\tau_{c}}{\sqrt{k_{c}}}\right)^{n+1} \frac{e_{c}^{n+1}-e_{c}^{n}}{\Delta t}-\left(\left|u^{n+1}\right|+\alpha\left|\frac{e_{c}^{n+1}-e_{c}^{n}}{\Delta t}\right|\right) U_{c}^{n+1}+n_{0} U_{0}\left|u^{n+1}\right|_{+} .
$$

\subsection{Positiveness of contractile variables}

The proposed scheme preserves the sign properties and the upper bound for the mesoscopic stiffness $k_{c}$ and for the local energy $\Psi_{c}$. Indeed, the local evolution equation for $k_{c}$ reads

$$
\left(1+\left|u^{n+1}\right| \Delta t+\alpha\left|e_{c}^{n+1}-e_{c}^{n}\right|\right) k_{c}^{n+1}=k_{c}^{n}+n_{0} k_{0}\left|u^{n+1}\right|_{+} \Delta t .
$$

Assuming $n_{0}$ constant and noting that $\left|u^{n+1}\right| \geq\left|u^{n+1}\right|_{+}$, this clearly implies that we have $0<k_{c}^{n+1}<n_{0} k_{0}$ as soon as $k_{c}^{n}$ satisfies the same inequalities, and this remains valid when $n_{0}$ is only "slowly-varying". As for the upper bound on $\tau_{c}^{2} / k_{c}$, it is obtained by multiplication of $(26)_{6}$ by $\left(\frac{\tau_{c}}{\sqrt{k_{c}}}\right)^{n+1}$ and subtraction of the result from (28). By observing that we have $U_{0} \geq \frac{\sigma_{0}^{2}}{2 k_{0}}$ and from the identity

$$
\left(\left(\frac{\tau_{c}}{\sqrt{k_{c}}}\right)^{n+1}-\left(\frac{\tau_{c}}{\sqrt{k_{c}}}\right)^{n}\right)\left(\frac{\tau_{c}}{\sqrt{k_{c}}}\right)^{n+1}=\frac{1}{2}\left(\left(\frac{\tau_{c}^{2}}{k_{c}}\right)^{n+1}-\left(\frac{\tau_{c}^{2}}{k_{c}}\right)^{n}\right)+\frac{1}{2}\left(\left(\frac{\tau_{c}}{\sqrt{k_{c}}}\right)^{n+1}-\left(\frac{\tau_{c}}{\sqrt{k_{c}}}\right)^{n}\right)^{2}
$$

this yields

$$
\begin{aligned}
\frac{1}{\Delta t}\left[\left(U_{c}^{n+1}\right.\right. & \left.\left.-\frac{1}{2}\left(\frac{\tau_{c}^{2}}{k_{c}}\right)^{n+1}\right)-\left(U_{c}^{n}-\frac{1}{2}\left(\frac{\tau_{c}^{2}}{k_{c}}\right)^{n}\right)\right]+\left(\left|u^{n+1}\right|+\alpha \frac{\left|e_{c}^{n+1}-e_{c}^{n}\right|}{\Delta t}\right)\left(U_{c}^{n+1}-\frac{1}{2}\left(\frac{\tau_{c}^{2}}{k_{c}}\right)^{n+1}\right) \\
& =n_{0}\left|u^{n+1}\right|_{+}\left(U_{0}-\frac{\sigma_{0}}{\sqrt{k_{c}^{n+1}}}\left(\frac{\tau_{c}}{\sqrt{k_{c}}}\right)^{n+1}+\frac{k_{0}}{2 k_{c}^{n+1}}\left(\frac{\tau_{c}^{2}}{k_{c}}\right)^{n+1}\right)+\frac{1}{2 \Delta t}\left(\left(\frac{\tau_{c}}{\sqrt{k_{c}}}\right)^{n+1}-\left(\frac{\tau_{c}}{\sqrt{k_{c}}}\right)^{n}\right)^{2} \\
& \geq \frac{1}{2} n_{0}\left|u^{n+1}\right|_{+}\left(\frac{\sigma_{0}}{\sqrt{k_{0}}}-\sqrt{\frac{k_{0}}{k_{c}^{n+1}}}\left(\frac{\tau_{c}}{\sqrt{k_{c}}}\right)^{n+1}\right)^{2} \geq 0,
\end{aligned}
$$

and we conclude like for $k_{c}$ that $\tau_{c}^{2} /\left(2 k_{c}\right)$ - namely, the energy $\Psi_{c}$ computed from the discrete variable - is bounded from above by $U_{c}$.

\subsection{Energy conservation}

As concerns the crucial issue of monitoring the evolution of the total energy of the system predicted by the discretization scheme (26), we can now prove the following result.

\section{Theorem 1}

By construction, the proposed time discretisation scheme (26) preserves the global energy dissipation properties of the original problem.

For the proof, we use the velocity related to the displacement increment rate by the trapezoidal rule

$$
\frac{\underline{v}^{n+1}+\underline{v}^{n}}{2}=\frac{\underline{y}^{n+1}-\underline{y}^{n}}{\Delta t}
$$

as test function $\underline{v}^{*}$ in the weak equilibrium equation $(26)_{2}$. We obtain

$$
\frac{\mathcal{K}^{n+1}-\mathcal{K}^{n}}{\Delta t}+\int_{\Omega_{0}} \underline{\underline{\Sigma}}^{n+\frac{1}{2} \sharp}: \mathrm{d}_{\underline{y}} \underline{e}^{n+\frac{1}{2} \sharp} \cdot \underline{v}^{n+\frac{1}{2}} d \Omega=\mathcal{P}_{\operatorname{ext}}^{n+\frac{1}{2}}\left(\underline{v}^{n+\frac{1}{2}}\right) .
$$


With the definition of $\mathrm{d}_{\underline{y}} \underline{e}^{n+\frac{1}{2} \sharp} \cdot \underline{v}^{n+\frac{1}{2}}$ we then notice that

$$
\begin{aligned}
\mathrm{d}_{\underline{y}} \underline{\underline{e}}^{n+\frac{1}{2} \sharp} \cdot \underline{v}^{n+\frac{1}{2}} & =\frac{1}{2}\left(\underline{\underline{F}}\left(\underline{\underline{y}}^{n+\frac{1}{2}}\right)^{T} \cdot \mathrm{d} \underline{\underline{y}} \underline{\underline{F}} \cdot \underline{v}^{n+\frac{1}{2}}+\left(\mathrm{d}_{\underline{y}} \underline{\underline{F}} \cdot \underline{v}^{n+\frac{1}{2}}\right)^{T} \cdot \underline{\underline{F}}\left(\underline{y}^{n+\frac{1}{2}}\right)\right) \\
& =\frac{1}{2}\left(\left(\frac{\underline{\underline{F}}^{n+1}+\underline{\underline{F}}^{n}}{2}\right)^{T} \cdot \frac{\underline{F}^{n+1}-\underline{\underline{F}}^{n}}{\Delta t}+\left(\frac{\underline{\underline{F}}^{n+1}-\underline{\underline{F}}^{n}}{\Delta t}\right)^{T} \cdot \frac{\underline{F}^{n+1}+\underline{\underline{F}}^{n}}{2}\right) \\
& =\frac{1}{2 \Delta t}\left(\left(\underline{\underline{F}}^{n+1}\right)^{T} \cdot \underline{\underline{F}}^{n+1}-\left(\underline{\underline{F}}^{n}\right)^{T} \cdot \underline{\underline{F}}^{n}\right)=\frac{\underline{\underline{e}}^{n+1}-\underline{\underline{e}}^{n}}{\Delta t}=\underline{\underline{e}}^{n+\frac{1}{2} \sharp} .
\end{aligned}
$$

Therefore, we have

$$
\underline{\underline{\underline{\Sigma}}}^{n+\frac{1}{2} \sharp}: \mathrm{d}_{\underline{y}} \underline{\underline{e}}^{n+\frac{1}{2} \sharp} \cdot \underline{v}^{n+\frac{1}{2}}=\left.\frac{\partial W_{e}}{\partial \underline{\underline{e}}}\right|^{n+\frac{1}{2} \sharp}: \frac{\underline{\underline{e}}^{n+1}-\underline{\underline{e}}^{n}}{\Delta t}+\left.\frac{\partial W_{v}}{\partial \underline{\underline{\dot{e}}}}\right|^{n+\frac{1}{2} \sharp}: \underline{\underline{e}}^{n+\frac{1}{2} \sharp}+\sigma_{1 \mathrm{D}}^{n+\frac{1}{2} \sharp}\left(\frac{e_{1 \mathrm{D}}^{n+1}-e_{1 \mathrm{D}}^{n}}{\Delta t}\right) .
$$

Let us start by analyzing the first two terms. By construction of the energy correction, the first term is exactly equal to the variation of mechanical energy per unit volume, namely, we have

$$
\left.\frac{\partial W_{e}}{\partial \underline{\underline{e}}}\right|^{n+\frac{1}{2} \sharp}: \frac{\underline{\underline{e}}^{n+1}-\underline{\underline{e}}^{n}}{\Delta t}=\frac{W_{e}^{n+1}-W_{e}^{n}}{\Delta t} .
$$

Then for the dissipative term we have by definition

$$
\left.\frac{\partial W_{v}}{\partial \underline{\underline{\dot{e}}}}\right|^{n+\frac{1}{2} \sharp}: \underline{\underline{e}}^{n+\frac{1}{2} \sharp}=\frac{\partial W_{v}}{\partial \underline{\underline{\dot{e}}}}\left(\underline{\underline{e}}^{n+\frac{1}{2} \sharp}\right): \underline{\underline{\underline{e}}}^{n+\frac{1}{2} \sharp} \geq 0 .
$$

It remains to handle the last term specific to the cardiac formulation. First, let us reformulate the time discretization of the rheological model, namely,

$$
\begin{aligned}
\frac{e_{1 \mathrm{D}}^{n+1}-e_{1 \mathrm{D}}^{n}}{\Delta t} & =\frac{\left(1+2 e_{s}^{n+1}\right)\left(1+2 e_{c}^{n+1}\right)-\left(1+2 e_{s}^{n}\right)\left(1+2 e_{c}^{n}\right)}{2 \Delta t} \\
& =\frac{e_{c}^{n+1}-e_{c}^{n}}{\Delta t}+\frac{e_{s}^{n+1}-e_{s}^{n}}{\Delta t}+\frac{2}{\Delta t}\left(e_{c}^{n+1} e_{s}^{n+1}-e_{c}^{n} e_{s}^{n}\right) \\
& =\left(1+2 e_{s}^{n+\frac{1}{2}}\right) \frac{e_{c}^{n+1}-e_{c}^{n}}{\Delta t}+\left(1+2 e_{c}^{n+\frac{1}{2}}\right) \frac{e_{s}^{n+1}-e_{s}^{n}}{\Delta t}
\end{aligned}
$$

where we have used the identity

$$
\begin{aligned}
\left(1+2 e_{s}^{n+\frac{1}{2}}\right) \frac{e_{c}^{n+1}-e_{c}^{n}}{\Delta t}+\left(1+2 e_{c}^{n+\frac{1}{2}}\right) \frac{e_{s}^{n+1}-e_{s}^{n}}{\Delta t} & = \\
\frac{e_{c}^{n+1}-e_{c}^{n}}{\Delta t}+\frac{e_{s}^{n+1}-e_{s}^{n}}{\Delta t} & +\frac{2}{\Delta t}\left(e_{c}^{n+1} e_{s}^{n+1}-e_{c}^{n} e_{s}^{n}\right) \\
& +\frac{1}{\Delta t} \underbrace{\left(e_{s}^{n} e_{c}^{n+1}-e_{s}^{n+1} e_{c}^{n}+e_{c}^{n} e_{s}^{n+1}-e_{c}^{n+1} e_{s}^{n}\right)}_{=0} .
\end{aligned}
$$

Therefore, using the stress relation in the rheological model we finally find

$$
\sigma_{1 \mathrm{D}}^{n+\frac{1}{2} \sharp}\left(\frac{e_{1 \mathrm{D}}^{n+1}-e_{1 \mathrm{D}}^{n}}{\Delta t}\right)=\sigma_{s}^{n+\frac{1}{2} \sharp}\left(\frac{e_{s}^{n+1}-e_{s}^{n}}{\Delta t}\right)+\sigma_{c}^{n+\frac{1}{2} \sharp}\left(\frac{e_{c}^{n+1}-e_{c}^{n}}{\Delta t}\right) .
$$

We can then focus on each term of this identity. For the series element, we directly have

$$
\sigma_{s}^{n+\frac{1}{2} \sharp}\left(\frac{e_{s}^{n+1}-e_{s}^{n}}{\Delta t}\right)=\left(\frac{E_{s}\left(e_{s}^{n+1}\right)^{2}-E_{s}\left(e_{s}^{n}\right)^{2}}{\Delta t}\right) .
$$


As concerns the active element, on the one hand we have by construction of $\sigma_{c}^{n+\frac{1}{2} \sharp}$

$$
\sigma_{c}^{n+\frac{1}{2} \sharp}\left(\frac{e_{c}^{n+1}-e_{c}^{n}}{\Delta t}\right)=\sqrt{k_{c}^{n+1}}\left(\frac{\tau_{c}}{\sqrt{k_{c}}}\right)^{n+1}\left(\frac{e_{c}^{n+1}-e_{c}^{n}}{\Delta t}\right)+\mu\left(\frac{e_{c}^{n+1}-e_{c}^{n}}{\Delta t}\right)^{2},
$$

and on the other hand, from the auxiliary energy equation

$$
\sqrt{k_{c}^{n+1}}\left(\frac{\tau_{c}}{\sqrt{k_{c}}}\right)^{n+1}\left(\frac{e_{c}^{n+1}-e_{c}^{n}}{\Delta t}\right)=\frac{U_{c}^{n+1}-U_{c}^{n}}{\Delta t}-n_{0} U_{0}\left|u^{n+1}\right|_{+}+\left(\left|u^{n+1}\right|+\alpha \frac{\left|e_{c}^{n+1}-e_{c}^{n}\right|}{\Delta t}\right) U_{c}^{n+1} .
$$

Altogether, we exactly recover the discretized version of the expected energy balance (25)

$$
\begin{aligned}
\frac{\mathcal{K}^{n+1}-\mathcal{K}^{n}}{\Delta t}+\frac{\mathcal{E}_{e}^{n+1}-\mathcal{E}_{e}^{n}}{\Delta t}+\int_{\Omega_{0}}\left(\frac{E_{s}\left(e_{s}^{n+1}\right)^{2}-E_{s}\left(e_{s}^{n}\right)^{2}}{\Delta t}+\frac{U_{c}^{n+1}-U_{c}^{n}}{\Delta t}\right) d \Omega= \\
\quad \mathcal{P}_{\operatorname{ext}}^{n+\frac{1}{2}}\left(\underline{v}^{n+\frac{1}{2}}\right)+\int_{\Omega_{0}} n_{0} U_{0}\left|u^{n+1 / 2}\right|_{+} d \Omega \\
\quad-\int_{\Omega_{0}}\left(\left|u^{n+1}\right|+\alpha \frac{\left|e_{c}^{n+1}-e_{c}^{n}\right|}{\Delta t}\right) U_{c}^{n+1} d \Omega-\int_{\Omega_{0}}\left(\mu\left(\frac{e_{c}^{n+1}-e_{c}^{n}}{\Delta t}\right)^{2}+\left.\frac{\partial W_{v}}{\partial \underline{\underline{e}}}\right|^{n+\frac{1}{2}}: \underline{\underline{e}}^{n+\frac{1}{2} \sharp}\right) d \Omega .
\end{aligned}
$$

Note that - as at the continuous level - this inequality is only informative when combined with the fact that - also at the discrete level $-U_{c}$ controls $\frac{\tau_{c}^{2}}{2 k_{c}}$ and that $0 \leq k_{c} \leq k_{0}$, because this shows that the growth of the fiber stress $\tau_{c}$ is controlled, in particular. Hence, all the physical variables of interest are controlled by this energy balance.

\section{Remark 6 (Incompressibility treatment)}

When the material considered is nearly-incompressible, we usually have a hyperelastic potential in the form

$$
W_{e}\left(J_{1}, J_{2}, J\right)=\bar{W}_{e}\left(J_{1}, J_{2}\right)+W_{\mathrm{vol}}(J-1),
$$

for an isotropic constitutive law, where $W_{\text {vol }}$ penalizes deformations such that $J-1 \neq 0$. The time discretization (27) can then be used as is with this particular expression. In case we want to represent an exactly incompressible material, a natural extension is then

$$
\underline{\underline{\underline{\Sigma}}}^{n+\frac{1}{2} \sharp}=\left.\frac{\partial \bar{W}_{e}}{\partial \underline{\underline{e}}}\right|^{n+\frac{1}{2} \sharp}+\left.\frac{\partial W_{v}}{\partial \underline{\underline{\dot{e}}}}\right|^{n+\frac{1}{2} \sharp}+\sigma_{1 \mathrm{D}}^{n+\frac{1}{2} \sharp} \underline{\tau}_{1} \otimes \underline{\tau}_{1}+\underline{\underline{\Sigma}}_{\mathrm{vol}}^{n+\frac{1}{2} \sharp},
$$

where $\underline{\underline{\Sigma}}_{\mathrm{vol}}^{n+\frac{1}{2} \sharp}$ accounts for the pressure contribution - in the continuous formulation $-p J \underline{\underline{C}}^{-1}$ - discretized here as

$$
\underline{\underline{\Sigma}}_{\mathrm{vol}}^{n+\frac{1}{2} \sharp}=-p^{n+\frac{1}{2} \sharp}\left(J \underline{\underline{C}}^{-1}\right)^{n+\frac{1}{2} \sharp}+p^{n+\frac{1}{2} \sharp}\left(J \underline{\underline{C}}^{-1}\right)^{n+\frac{1}{2} \sharp}: \underline{\underline{\dot{e}}}^{n+\frac{1}{2} \sharp} \frac{\underline{\underline{e}}^{n+\frac{1}{2} \sharp}}{\underline{\underline{e}}^{n+\frac{1}{2} \sharp}: \underline{\underline{e}}^{n+\frac{1}{2} \sharp}},
$$

in which $\left(J \underline{\underline{C}}^{-1}\right)^{n+\frac{1}{2} \sharp}=\left(J \underline{\underline{C}}^{-1}\right)\left(\underline{y}^{n+\frac{1}{2}}\right)$, and $p^{n+\frac{1}{2} \sharp}$ is an actual unknown related to the Lagrange multiplier of the incompressibility constraint

$$
J\left(\underline{y}^{n+1}\right)=1 .
$$

Note that we have the property

$$
\underline{\underline{\Sigma}}_{\mathrm{vol}}^{n+\frac{1}{2} \sharp}: \underline{\underline{\dot{e}}}^{n+\frac{1}{2} \sharp}=0,
$$

hence, the pressure contribution does not produce any work on the actual (discrete) strain rate, as desired. 


\subsection{Spatial discretization}

In order to complete the discretization of the problem, we choose a finite element displacement space $V_{h}$ and we seek $\left(\underline{y}_{h}, \underline{v}_{h}\right) \in\left(V_{h}\right)^{2}$ which satisfy (26) for test functions $\underline{v}^{*} \in V_{h}$. Note that this does not alter the energy conservation property. As regards numerical integration, its impact on the discrete energy balance is that a very similar conservation identity holds with weighted sums substituted for integrals. Then, provided the numerical integration scheme considered uses only positive weights, the production (positive) and dissipation (negative) terms in the right-hand side of this balance equation preserve their interpretations. Of course, in practice the variables $\left(e_{c}, k_{c}, \tau_{c} / \sqrt{k_{c}}\right)$ - and optionally $U_{c}$ - need only be solved for at the integration points, and they can be eliminated at the element level by a Schur complement procedure, hence they do not increase the size of the linear systems to be solved [31].

As regards incompressible or nearly-incompressible formulations, some caution must be exercised in order to avoid numerical locking. This difficulty is usually handled by substituting

$$
W_{\mathrm{vol}}^{h}(J-1)=W_{\mathrm{vol}}\left(\Pi_{h}(J-1)\right),
$$

for $W_{\text {vol }}$ in the hyperelastic potential, where $\Pi_{h}$ represents a (spatial) projection operator typically according to the $L^{2}$ dot product - onto a "discrete pressure space" of low polynomial order. This corresponds to a mixed formulation in which the pressure is considered as an additional unknown [5]. Indeed, the incompressibility constraint that tends to prevail is then $\Pi_{h}(J-1)=0$, which is more adapted to finite element displacements than the original constraint $J=1$, provided $\Pi_{h}$ is adequately chosen in accordance with $V_{h}$. Together with the time discretization, this leads to the following pressure contribution

$$
\begin{aligned}
\underline{\underline{\Sigma}}_{\mathrm{vol}, h}^{n+\frac{1}{2} \sharp}= & -p_{h}^{n+\frac{1}{2} \sharp}\left(J \underline{\underline{C}}^{-1}\right)^{n+\frac{1}{2} \sharp} \\
& +\left[W_{\mathrm{vol}}^{h}\left(J^{n+1}-1\right)-W_{\mathrm{vol}}^{h}\left(J^{n}-1\right)+p_{h}^{n+\frac{1}{2} \sharp}\left(J \underline{\underline{C}}^{-1}\right)^{n+\frac{1}{2} \sharp}: \underline{\underline{\dot{e}}}^{n+\frac{1}{2} \sharp} \frac{\underline{\underline{e}}^{n+\frac{1}{2} \sharp}}{\underline{\underline{e}}^{n+\frac{1}{2} \sharp}: \underline{\underline{e}}^{n+\frac{1}{2} \sharp}},\right.
\end{aligned}
$$

where $p_{h}^{n+\frac{1}{2} \sharp}$ is tied to the displacement - for a nearly-incompressible formulation - by

$$
p_{h}^{n+\frac{1}{2} \sharp}=-\Pi_{h}\left(W_{\mathrm{vol}}^{\prime}\left(\Pi_{h}\left(J\left(\underline{y}_{h}^{n+\frac{1}{2}}\right)-1\right)\right)\right) .
$$

When the material considered is exactly incompressible, this simplifies into

$$
\underline{\underline{\Sigma}}_{\mathrm{vol}, h}^{n+\frac{1}{2} \sharp}=-p_{h}^{n+\frac{1}{2} \sharp}\left(J \underline{\underline{C}}^{-1}\right)^{n+\frac{1}{2} \sharp}+p_{h}^{n+\frac{1}{2} \sharp}\left(J \underline{\underline{C}}^{-1}\right)^{n+\frac{1}{2} \sharp}: \underline{\underline{\underline{e}}}^{n+\frac{1}{2} \sharp} \frac{\underline{\underline{e}}^{n+\frac{1}{2} \sharp}}{\underline{\underline{\dot{e}}}^{n+\frac{1}{2} \sharp}: \underline{\underline{e}}^{n+\frac{1}{2} \sharp}},
$$

as in the continuous formulation, and $p_{h}^{n+\frac{1}{2} \sharp}$ then represents an additional unknown associated with the constraint

\section{Remark 7}

Determining the actual compressibility level of living tissues is still a largely open question. This is - indeed - one among the many uncertainties to be confronted when simulating and validating models of living systems. Therefore, a possible approach regarding incompressibility treatment is to adjust the bulk modulus so that the volume changes are only limited to about $5 \%$ - a level of accuracy well-acceptable in this framework - in which case numerical locking is of no major concern in practice. Thus, resorting to mixed procedures can be reserved to situations in which incompressibility must be strictly enforced, and when a very high accuracy is expected. 


\section{Concluding remarks}

We have revisited an earlier-proposed muscle tissue model and presented a complete analysis - based on energy considerations - of a refined version of this model allowing to account for energy inflows. To that purpose we have introduced the new energy variable $U_{c}$ representing the microscopic elastic energy stored in the sarcomeres. This variable is distinct from the macroscopic elastic energy $\Psi_{c}$, but we have established that it is the natural quantity to be considered in energy balances, and furthermore that it controls the macroscopic energy.

We then proceeded to propose a discretization strategy - in time and space - which entirely preserves the derived energy balances, including the positiveness of the discrete microscopic energy. This led us to specifying in details the time scheme, whereas more flexibility is allowed in the choice of the space discretization (finite element shape functions), provided positive weights are used in the integration rule and adequate caution is exercised to avoid numerical locking when incompressible formulations are considered.

This type of analysis will be crucial in order to adequately model energy transfers in the complete chain originating from oxygen supply in the blood and resulting in the production of mechanical work in the muscles, with the many important physiological - normal and pathological - phenomena associated with this energy flow. This is a natural perspective to the present work, indeed, and the topic of some future papers by the authors.

\section{References}

[1] R. Balaban. The role of $\mathrm{Ca}^{2+}$ signaling in the coordination of mitochondrial ATP production with cardiac work. Biochimica et Biophysica Acta (BBA) - Bioenergetics, 1787(11):1334-1341, 2009.

[2] D. Balzani, P. Neff, J. Schröder, and G. Holzapfel. A polyconvex framework for soft biological tissues. adjustment to experimental data. International Journal of Solids and Structures, 43:6052-6070, 2006.

[3] J. Bestel. Modèle différentiel de la contraction musculaire contrôlée. Application au système cardio-vasculaire. PhD thesis, University Paris IX Dauphine, 2000.

[4] J. Bestel, F. Clément, and M. Sorine. A biomechanical model of muscle contraction. In Lecture Notes in Computer Science, volume 2208. Eds W.J. Niessen and M.A. Viergever, Springer-Verlag, 2001.

[5] F. Brezzi and M. Fortin. Mixed and Hybrid Finite Element Methods. Springer-Verlag, 1991.

[6] R. Chabiniok, D. Chapelle, P. Lesault, A. Rahmouni, and J. Deux. Validation of a biomechanical heart model using animal data with acute myocardial infarction. In CI2BM09 MICCAI Workshop on Cardiovascular Interventional Imaging and Biophysical Modelling, London United Kingdom, 2009.

[7] D. Chapelle, F. Clément, F. Génot, P. Le Tallec, M. Sorine, and J. Urquiza. A physiologically-based model for the active cardiac muscle. In Lectures Notes in Computer Science, volume 2230. Eds T. Katila, I.E. Magnin, P. Clarysse, J. Montagnat, J. Nenonen, Springer-Verlag, 2001.

[8] D. Chapelle, M. Fernàndez, J.-F. Gerbeau, P. Moireau, J. Sainte-Marie, and N. Zemzemi. Numerical simulation of the electromechanical activity of the heart. In Proceedings of 
Functional Imaging and Modeling of the Heart 2009 (FIMH'09), volume 5528 of LNCS, pages 239-248, June 2009.

[9] P. Ciarlet and G. Geymonat. Sur les lois de comportement en élasticité non linéaire. C.R.A.S, Série II, 295:423-426, 1982.

[10] K. Costa, J. Holmes, and A. McCulloch. Modeling cardiac mechanical properties in three dimensions. Phil. Trans. R. Soc. Lond. A, 359:1233-1250, 2001.

[11] Y. Fung. Biomechanics: Mechanical Properties of Living Tissues. Springer, 2nd edition, 1993.

[12] S. Göktepe, S. Acharya, J. Wong, and E. Kuhl. Computational modeling of passive myocardium. Commun. Numer. Meth. Engng, 27(1):1-12, 2011.

[13] O. Gonzales. Exact energy and momentum conserving algorithm for general models in nonlinear elasticity. Comput. Meth. Appl. Mech. Eng., 190(13-14):1763-1783, 2000.

[14] P. Hauret and P. Le Tallec. Energy controlling time integration methods for nonlinear elastodynamics and low velocity impact. Comput. Meth. Appl. Mech. Eng., 195:4890-4916, 2006 .

[15] V. Hill. The heat of shortening and the dynamic constants of muscle. In Proc. Roy. Soc. London, volume 126 of Series B, pages 126-136, 1938.

[16] G. Holzapfel and R. Ogden. Constitutive modelling of passive myocardium: a structurally based framework for material characterization. Phil. Trans. R. Soc. A, 367:3445-3475, 2009 .

[17] J. Humphrey. Cardiovascular Solid Mechanics - Cells Tissues and Organs. Springer, 2002.

[18] J. Humphrey. Continuum biomechanics of soft tissues. Proc. R. Soc. Lond. A, 459:3-46, 2002.

[19] P. Hunter, A. McCulloch, and H. ter Keurs. Modelling the mechanical properties of cardiac muscle. Progr. Biophys. Mol. Biol., 69:289-331, 1998.

[20] A. Huxley. Muscle structure and theories of contraction. In Progress in biophysics and biological chemistry, volume 7, pages 255-318. Pergamon press, 1957.

[21] P. Krejci, J. Sainte-Marie, M. Sorine, and J. Urquiza. Solutions to muscle fiber equations and their long time behaviour. Nonlinear Analysis: Real World Applications, 7(4):535-558, September 2006.

[22] P. Le Tallec. Numerical Analysis of Viscoelastic Problems, volume 15 of Recherches en Mathématiques Appliquées. Masson, 1990.

[23] P. Le Tallec. Numerical methods for nonlinear three-dimensional elasticity. In P. G. Ciarlet and J.-L. Lions, editors, Handbook of Numerical Analysis, volume 3. Elsevier, 1994.

[24] P. Le Tallec and P. Hauret. Energy conservation in fluid structure interactions. In Y. Kuznetsov, P. Neittanmaki, and O. Pironneau, editors, Numerical Methods for Scientific Computing - Variational Problems and Applications, 2003.

[25] I. Mirsky and W. Parmley. Assessment of passive elastic stiffness for isolated heart muscle and the intact heart. Circulation Research, XXXIII:233-243, 1973. 
[26] P. Moireau. Filtering based data assimilation for second order hyperbolic PDEs. Applications in cardiac mechanics. PhD thesis, Ecole Polytechnique, 2008.

[27] M. Nash and P. Hunter. Computational mechanics of the heart - from tissue structure to ventricular function. Journal of Elasticity, 61:113-141, 2000.

[28] P. Nelson. Biological Physics: Energy, Information, Life. W.H. Freeman \& Company, 2003.

[29] A. Raoult. Symmetry groups in nonlinear elasticity: An exercise in vintage mathematics. Comm. on Pure Appl. Anal., 8(1):435-456, 2009.

[30] R. Rivlin and J. Ericksen. Stress-deformation relations for isotropic materials. J. Rational Mech. Anal., 4:323-425, 1955.

[31] J. Sainte-Marie, D. Chapelle, R. Cimrman, and M. Sorine. Modeling and estimation of the cardiac electromechanical activity. Computers \& Structures, 84:1743-1759, 2006.

[32] V. Saks, R. Favier, R. Guzun, U. Schlattner, and T. Wallimann. Molecular system bioenergetics: regulation of substrate supply in response to heart energy demands. The Journal of Physiology, 577(3):769-777, 2006.

[33] D. Veronda and R. Westmann. Mechanical characterization of skin-finite deformation. Journal of Biomechanics, 3:114-124, 1970. 